\title{
A synthesis of approaches to upscaling of hydraulic conductivities
}

\author{
Xavier Sánchez-Vila \\ Departament Matemàtica Aplicada III, Escola Tècnica Superior d'Enginyers de Camins \\ Universitat Politècnica de Catalunya, Barcelona, Spain \\ Jorge P. Girardi \\ Consejo Nacional de Investigaciones Científicas y Técnicas, Universidad Nacional de San Juan \\ San Juan, Argentina

\section{Jesús Carrera} \\ Departament d'Enginyeria del Terreny, Escola Tècnica Superior d'Enginyers de Camins \\ Universitat Politècnica de Catalunya, Barcelona, Spain
}

\begin{abstract}
Simulation of flow through heterogeneous media often requires discretizing the flow domain into blocks and assigning an equivalent block conductivity value to each one of them. The process of defining block conductivities from point values is termed upscaling. A number of approaches to upscaling are available, most of which consider the uncertainty associated with any natural property, so that they cast the problem in a stochastic frame. Recently, Indelman and Dagan (1993a, b) provided a general stochastic methodology to upscaling in heterogeneous anisotropic formations by means of the dissipation energy function; unfortunately, they did not provide any "practical" method to compute block values from point ones. The objective of this work is twofold: First, we analyze different practical approaches to compute block conductivities and find that all of them provide very similar results in terms of actual computed values; second, we check that all approaches verify approximately a number of conditions stated by Indelman and Dagan (1993a). Specifically, we show analytically that for regular blocks, the methodologies of both Rubin and Gómez-Hernández (1990) and Desbarats (1992) (which we call "practical" methodologies) satisfy the condition that the effective conductivity obtained from a field where the elementary conductivities are defined over a certain support (we call this the actual formation) is identical to that obtained from the same field with conductivities defined at a larger support (upscaled formation). The analysis is carried out by working with the logarithm of block conductivities and using a smallperturbation expansion and thus is strictly valid for small variances. On the other hand, we show numerically that the two methodologies satisfy approximately an important condition stated in terms of the dissipation energy: that block-averaged dissipation values computed are indeed very close to the true dissipation values in each block. The agreement is even better if we consider statistical moments instead of point values. As an important conclusion we should note that all practical methodologies considered in this work perform equally well and, more important, constitute a simple way to treat an otherwise very complex problem.
\end{abstract}

\section{Introduction}

Heterogeneity is one of the main characteristics associated with many variables in nature. It is also one of the most difficult aspects in modeling groundwater flow and solute transport, both in porous and fractured media. One way of taking into account the complexity of the natural media would be to build deterministic models which incorporate natural heterogeneity in an explicit manner. Because of limitations on available data, this approach is not practical in real cases. An alternative is to consider natural variables as stochastic processes, character-

Copyright 1995 by the American Geophysical Union.

Paper number 94WR02754

0043-1397/95/94WR-02754\$05.00 ized by a few statistical parameters. By using this approach, partial differential equations describing groundwater flow and solute transport become stochastic differential equations. Solutions for the dependent variables are given also in statistical terms.

One of the questions that arise in groundwater problems is how to treat heterogeneity in large-scale aquifer models. In some cases we are interested not in accurately reproducing aquifer behavior at the local scale, but in studying variables at some higher (maybe regional) scale. For that purpose it is important to find equivalent parameters, that is, those which reproduce the average behavior of the system discretized at a certain scale. This scale need not necessarily be the same at which we have obtained our measurements (data support). It is also important to notice that the average behavior at a given 
scale is not reproduced by arithmetically averaging local values of aquifer parameters.

The problem of finding equivalent parameters is termed upscaling, because it involves increasing the size of the domain over which they are defined. The most important reason for upscaling is the necessity of incorporating measurements taken at different supports (scales of observation). Another reason is a very practical one: Numerical solutions involve partition of the aquifers into meshes whose elements cannot be smaller than a certain size (in order to be handled by present available computers); unfortunately, this size is generally larger than the scale of our measurements. A closely related problem, frequently addressed in the literature, is the determination of effective properties in heterogeneous media. Effective parameters are defined as representative values of the mean behavior through an ensemble of realizations, while equivalent parameters are associated with a certain geometry and defined as spatial averages computed on a single realization. These two definitions should converge to the same value for very large geometries and under the assumption of ergodicity.

When we partition the medium into blocks of a certain size, we face the following problem: Can we associate an equivalent value with each one of the blocks, and, if so, how can we compute it? Let us start by considering a large domain where a certain parameter (e.g., hydraulic conductivity) is defined at each point. Flow takes place in this domain under natural conditions. Now, if we partition the domain into blocks, we

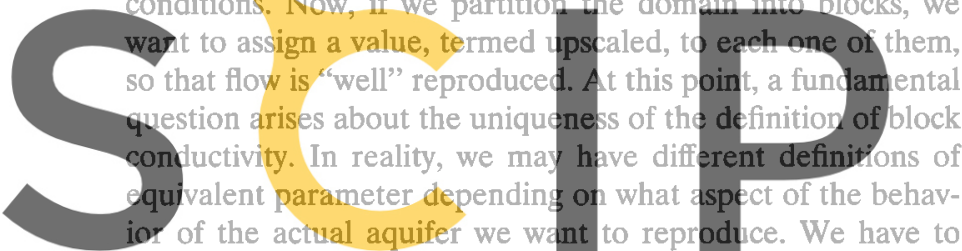
acknowledge that upscaling will lead to a loss of information regarding the local scale, as we eliminate heterogeneity at

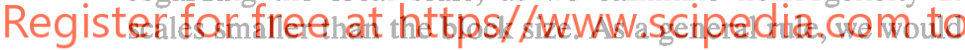
like to reproduce the global behavior of the aquifer, while keeping the local behavior as close as possible to reality. Let us start by considering a certain parallelepipedic domain (block) inside a larger aquifer. We can extract this block and apply a head difference $(\Delta H)$ between two opposite faces (two lines in two dimensions), while applying a no-flow condition to the remaining boundaries. If the total flow through the block is $Q$, it is easy to define a block permeability, $K_{b}=Q / \Delta H$, which reproduces the global behavior. The main problem is that this definition is linked to the choice of boundary conditions, so that when we put the block back into the aquifer, the real boundary conditions are not as simple. Some alternative definition must be sought.

Indelman and Dagan [1993a, b] proposed a definition for block permeability $\left(\mathbf{K}_{V}\right)$ based on the concept of energy dissipation. They defined $\mathbf{K}_{V}$ as a tensor which satisfies the following property: Energy dissipated at the block under natural boundary conditions is equal to the volumetric average of energy dissipated throughout the block. This definition is physically correct, but mathematically difficult. The main problem arises from the fact that for a single flow field, one cannot derive the full tensor for each block from the exhaustive point $K$ distribution. The reason is that the number of unknowns exceeds the number of equations. For that matter, equality can only be established in statistical terms (i.e., the first two statistical moments of energy), and even so, in many cases the structure of the tensor must be postulated, in order to reduce the number of independent unknowns. This indeterminacy in the exhaustive information case can be eliminated by using a different approach, the one by Rubin and Gómez-Hernández [1990]. These authors selected a block in the real aquifer and, under natural conditions, computed average flow and average head gradient. They defined block permeability $\left(\mathbf{K}_{b}\right)$ as the tensor that relates both averages. This system cannot provide a unique solution, as the number of unknowns exceeds the number of equations. To avoid this problem, the authors assumed $K_{b}$ to be a scalar, so that flow and gradient volumetric averages are colinear. This methodology reproduces well the behavior of the whole aquifer, but at the same time provides a good representation of piezometric heads, whenever natural conditions are kept. It is not clear, however, that the same value would be obtained when these conditions are changed.

From the previous paragraph, it is clear that a simple solution cannot be found for the general case. In many situations, however, we deal with less complex problems, where the medium can be considered statistically isotropic (this is particularly true in two-dimensional media) and we partition it into blocks of regular shape. In this case, although we recognize that block permeability must still be defined as a tensorial parameter, we assert that for a statistically isotropic medium where blocks are either squares (in two dimensions) or cubes (in three dimensions) the tensor can be substituted by a scalar,

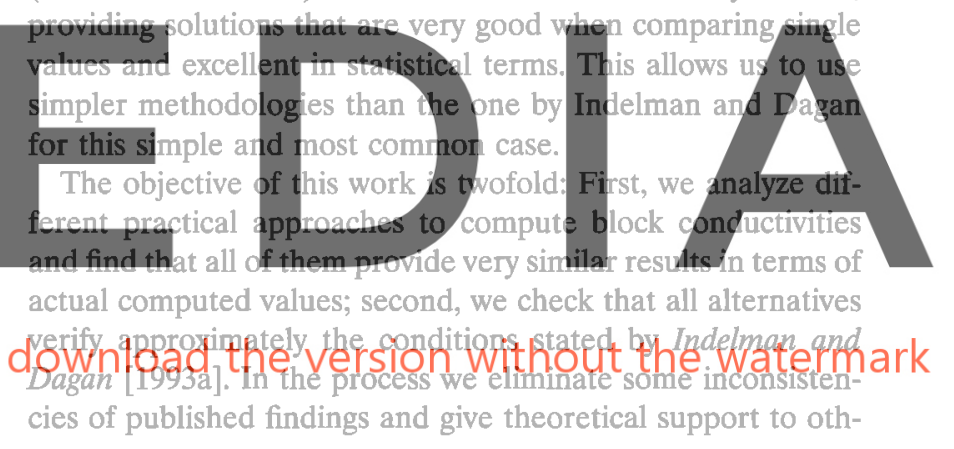
ers.

The paper is structured as follows: Section 2 presents a synthesis of different formulations to upscaling that appear in the literature, and their similarities are pointed out. Section 3 is devoted to deriving analytically the statistical moments of log block conductivity based on the approach by Rubin and Gomez-Hernández [1990]. These moments are analyzed for the two- and three-dimensional cases separately. Specifically, we show analytically that the approaches of both Rubin and Gómez-Hernández [1990] and Desbarats [1992] satisfy the condition that the effective conductivities of upscaled and actual formations are identical. Moreover, the moments of log block conductivities compare well with those given by other methodologies. This is demonstrated using a small-perturbation expansion, and thus it is strictly valid for small variances. Section 4 is devoted to numerical simulations in two dimensions which are used also to check analytical results from section 3 . They are found valid at least up to point log conductivity variances equal to 1.0 . Section 5 is entirely devoted to the numerical analysis of the dissipation function for different approaches. We show numerically that block-averaged dissipation values computed by practical methods are indeed very close to the true values. The agreement is even better if we consider statistical moments instead of point values. Finally, a summary and some conclusions are stated. 


\section{Review of Different Formulations of Upscaling: Conjectures}

Literature in equivalent parameters is closely related to a problem which has been studied much longer: finding effective parameters for saturated flow. We must keep in mind that under ergodicity, effective and equivalent values should converge to a unique solution for very large blocks. This is an important property because it provides a check to any study on equivalent parameters, as the limiting values for very large blocks can be obtained independently. Deriving effective parameters is a classical problem that has been addressed by several authors since Matheron [1967], who quoted previous work from the Russian literature by Schvidler [1962]. Their work was carried out using analytical perturbation methods in infinite, ergodic, isotropically correlated fields. Their main result was that for constant mean parallel flow in a twodimensional infinite domain with stationary random function $K$ of isotropic correlation structure, the effective hydraulic conductivity $K_{\text {ef }}$ was equal to the geometric mean of point values, $K_{G}$. Gutjahr et al. [1978] expanded this work to one and three dimensions. Gelhar and Axness [1983] included anisotropic correlation structures. Dagan [1982] obtained similar values using a self-consistent approach. Others have relaxed some of the assumptions made by the above authors. Dagan [1982] and Kitanidis [1990] have studied gradually varying mean flow. Loaiciga et al. [1993] considered the presence of trends in mean $K$. Naff [1991] analyzed radial flow. Neuman and Om [1993] carried out a very comprehensive study where anrong many other things, they analyze the existence

bounded domains, by explicitly

conditions. Their work has been

nd S. P. Neuman (The effect of flow, submitted to Water Resources Research, 1994) for the particular case of constant head boundaries. After Smith and

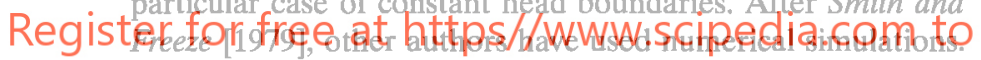
For example, Desbarats [1987] analyzed effective permeability in sand-shale formations. More recently, a large number of authors, since Joumel et al. [1986], have used a combined analytical-numerical method to estimate effective parameters based on a power-averaging formula. Gómez-Hernández and Gorelick [1989] used this type of formula to analyze a synthetic case. Ababou and Wood [1990] found the power-averaged value as a function of the different means of $K$ : geometric, arithmetic, and harmonic. Desbarats [1992] analyzed the validity of the formula for high variances. Ababou [1993] applied it for anisotropic heterogeneous formations.

The literature in equivalent (block) parameters is more recent and, certainly, not as broad. A review of this literature provides us with a number of different approaches to upscaling that will be compared throughout the text. For that reason, in this section we summarize the most important points of each of them, so that the frame in which the rest of the work is carried out remains clear from the very beginning. Although we acknowledge the contributions of many authors to some of the approaches, we will denote each of them by the name(s) of the principal author(s). The order in which the approaches appear is strictly chronological.

\subsection{Approach 1: Darcian Definition}

This approach is outlined in Figure 1. It was introduced in the previous section and consists of isolating the block, impos-

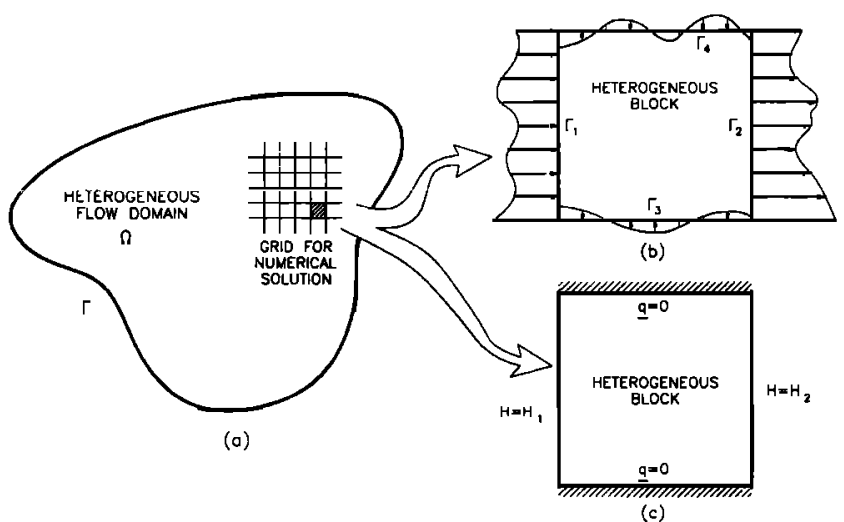

Figure 1. Derivation of block conductivities. The approaches of Rubin and Gómez-Hernández [1991] and of Indelman and Dagan [1993] are based on studying the block in Figure 1b under the same conditions of the regional flow domain (Figure 1a). The Darcian approach is based on applying the boundary conditions indicated in Figure 1c.

ing a uniform gradient $\left(\left(H_{2}-H_{1}\right) / L_{x}\right)$, and computing the flow rate across it, using the true values of point conductivity. Block conductivity (transmissivity, actually, in a twodimensional medium such as the one in the figure) would then be defined as:

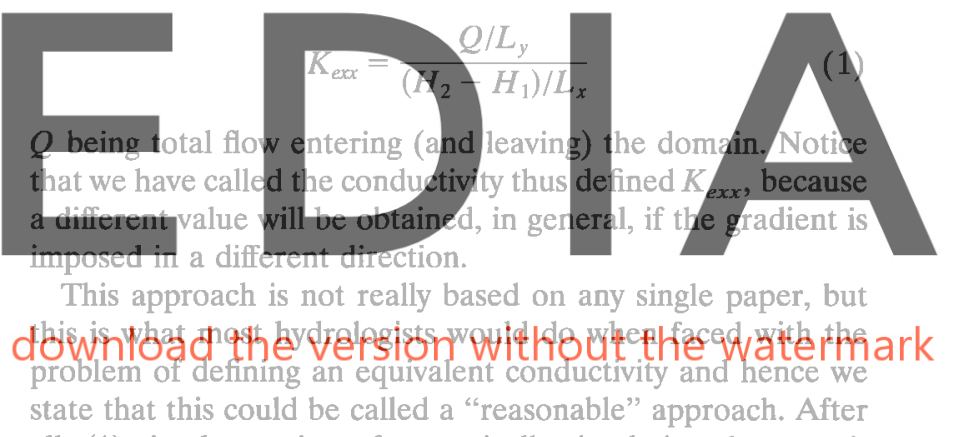

all, (1) simply consists of numerically simulating the experiment of Darcy, who first defined hydraulic conductivity.

With the exception of Gómez-Hernández [1990, 1991], who used an approach similar to (1) for defining interfacial transmissivities for finite differences models, no one has used this approach to derive theoretical results. This is probably a consequence of the difficulties associated with establishing the covariances between $K_{\text {exx }}$ and heads and point conductivities away from the block. In fact, Gómez-Hernández [1991] was forced to derive those covariances numerically, which requires much computer time. In this context, one should recall that the objective of this type of work is not only defining block $K$ from true point values, which are not known in a real case, but defining the statistics of block $K$, which allow practical geostatistical work.

A variation of this approach consists of substituting the no-flow boundaries by prescribed head boundaries. Along these, heads vary linearly, so that the gradient inside a homogeneous block would be constant. This variation may be required when dealing with anisotropic equivalent conductivities, something which falls beyond the scope of this work.

\subsection{Approach 2: Rubin and Gómez-Hernández [1990]}

Rubin and Gómez-Hernández [1990] provided the pioneering paper on the topic of block conductivity. By considering two- 
dimensional flow, isotropic covariance structure, and square blocks, they gave the classical definition of block conductivity (considered to be a scalar) as the ratio between the average local flow and average gradient in a block of a certain size, both of them computed in the direction of the expected head gradient value (their equation (5)). So, they defined block conductivity $K_{b}$ as

$$
K_{b} \int_{V} \frac{\partial h}{\partial x} d V=-\int_{V} q_{x} d V
$$

where $V$ is the block, $K_{b}$ block conductivity, $q_{x}$ local flux (Darcy's velocity) in the direction of the mean flow gradient, and $\partial h / \partial x$ piezometric head gradient in the same direction, where $h(\mathbf{x})$ is the solution of the flow problem, within the heterogeneous block, under uniform mean head gradient. The justification for this formula was not given, but if Darcy's law is averaged over the block, while assuming that average gradients in the exact and block-averaged domains are similar, then

$$
\begin{aligned}
& \overline{q_{x}}=K_{x x} \overline{j_{x}}+K_{x y} \overline{j_{y}} \\
& \overline{q_{y}}=K_{y x} \overline{j_{x}}+K_{y y} \overline{j_{y}}
\end{aligned}
$$

where $j_{x}=\partial h / \partial x$ and the overbar stands for block averages. If the mean gradient is uniform and in the $X$ direction, then symmetry considerations ensure $\left\langle K_{x y}\right\rangle=0$. This lends some port to the simplification of colinearity between q and $\nabla h$ is assumed in (2) fact, (2) was originally deriver Gómez-Hernández, 1990, e if average flux is parallel ors circumvented this problem in a vecto rial form [Rubin [1993], this need not be the case. Gómez-Hemández [1991] solved the problem by using a tensorial. block conductivity.

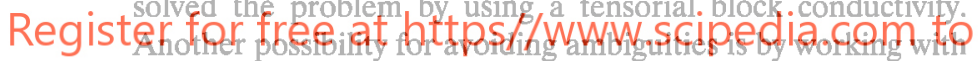
averages of projections over a given direction d. By multiplying both sides of the original equation in vectorial form by d, $K_{b}$ can be expressed as

$$
K_{b}=-\frac{\int_{V} \mathbf{q d} d V}{\int_{V} \nabla h \mathbf{d} d V}
$$

Now, taking $\mathbf{d}=(1,0)=\nabla x$, these two quantities can be simply obtained as

$$
\int_{V} \mathbf{q d} d V=\int_{\Gamma} x \mathbf{q n} d \Gamma=Q_{1} x_{1}+Q_{2} x_{2}+Q_{3} x_{G 3}+Q_{4} x_{G 4}
$$

$$
\int_{V} \nabla h \mathbf{d} d V=\int_{\Gamma} h \mathbf{d n} d \Gamma-\int_{V} h \nabla \cdot \mathbf{d} d V=\left(\bar{H}_{2}-\bar{H}_{1}\right) L_{y}
$$

where $Q_{t}=\int_{\Gamma_{1}}$ qn $d \Gamma$ is the flow rate across the $i$ th boundary and $x_{G l}$ is the center of gravity of the boundary fluxes (see Figure 1b). Rearranging terms and considering that $\Sigma Q_{t}=0$, we can rewrite (6) as

$\int_{V} \mathbf{q d} d V=\frac{Q_{1}-Q_{2}}{2} L_{x}+\frac{Q_{3}-Q_{4}}{2}\left(x_{G 3}-x_{G 4}\right)$

$$
+\left(Q_{3}+Q_{4}\right)\left(\frac{x_{G 3}+x_{G 4}}{2}-\frac{x_{1}+x_{2}}{2}\right)
$$

The last term should be small both because $Q_{3} \simeq-Q_{4}$ (what enters from the top will leave through the bottom in smooth fields) and because $\left(x_{G 3}+x_{G 4}\right)$ should be similar to $\left(x_{1}+\right.$ $\left.x_{2}\right)$. The second term can be neglected whenever $x_{G 3} \simeq x_{G 4}$. Under these conditions, which are expected in a field with moderate $\sigma_{Y}^{2}$ and uniform mean gradient in the $X$ direction, $K_{e x x}$ equals $K_{b}$.

For the flow conditions defined in section 2.1 and Figure 1c (Darcian approach), $Q_{3}=Q_{4}=0$ and $Q_{1}=-Q_{2}$. Therefore substituting (6) and (7) into (5) leads to a $K_{b}$ value identical to $K_{e x x}$ in (1). It is clear that under those conditions, the two definitions coincide.

Rubin and Gómez-Hernández [1990] noted that $K_{b}$ coincides with $K_{\text {eff }}$ for large $V$. By considering $K_{b}(\mathbf{x})$ to be a realization of an ergodic random function, they were able to derive the statistics (mean and covariance) of this function, both for the unconditional and conditional cases. Their work was expanded by Gómez-Hernández [1991], who assumed $K_{b}(\mathbf{x})$ to be a multiGaussian random function and used the previous derivations to simulate directly block conductivity values conditioned upon measurements at the local scale. In fact, the most important point about the method of Rubin and Gómez-Hernández is, precisely, the possibility of conditioning the block conductivities upon measurements taken with different supports.
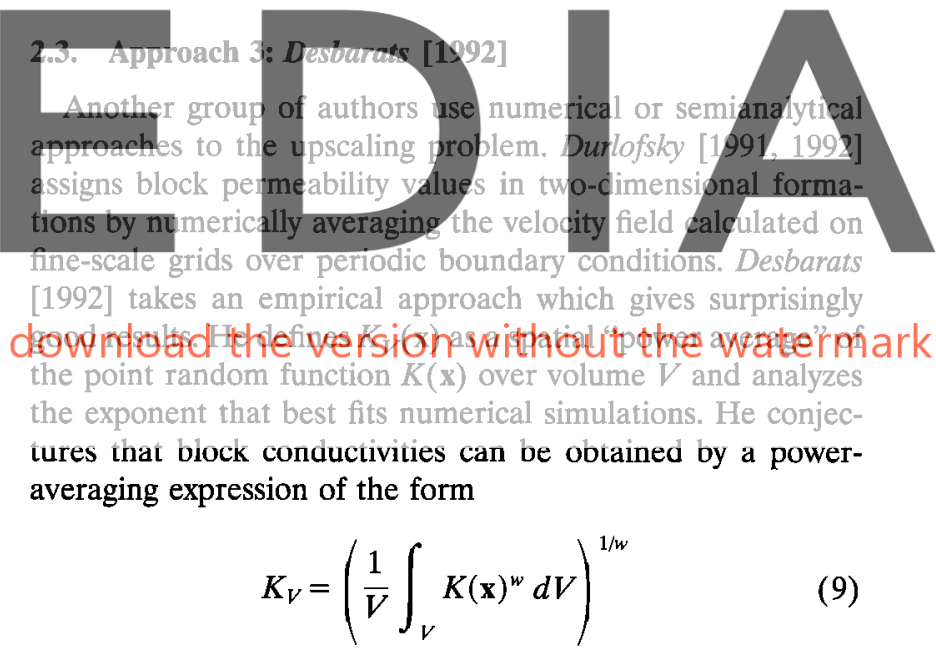

ditions. Desbarats 1992] takes an empirical approach which gives surprisingly outpthe aveatce'rphark Iume $V$ and analyzes the exponent that best fits numerical simulations. He conjectures that block conductivities can be obtained by a poweraveraging expression of the form

$$
K_{V}=\left(\frac{1}{V} \int_{V} K(\mathbf{x})^{w} d V\right)^{1 / w}
$$

where $w=0$ is equivalent to geometric averaging. The mean and variance of $Y_{V}=\ln K_{V}$ are linearly approximated by

$$
\begin{gathered}
\left\langle Y_{V}\right\rangle=\langle Y\rangle+(w / 2)\left(\sigma_{Y}^{2}-\overline{C_{Y}}\right) \\
\operatorname{Var}\left(Y_{V}\right)=\overline{C_{Y}}
\end{gathered}
$$

where

$$
\overline{C_{Y}}=\left(\int_{V} \int_{V} C_{Y}\left(\mathbf{x}-\mathbf{x}^{\prime}\right) d \mathbf{x} d \mathbf{x}^{\prime}\right) / V^{2}
$$

and $C_{Y}$ is the covariance function of $Y=\ln K$. The covariance function of $Y_{V}$ can also be derived in a simple manner. Based on numerical simulations, Desbarats showed that for threedimensional uniform gradient flow, and cubic blocks, $\omega=1 / 3$ leads to $K_{V}$ values very close to those obtained using (1) (an unequivocal sign that he also considers (1) to be reasonable). The simplicity of this approach makes it very appealing. More- 
over, his numerical proof seems unquestionable. For cubic blocks, isotropic covariance, and uniform mean gradient, Desbarats [1992] showed numerically that the definition of block conductivity by Rubin and Gómez-Hernández [1990] (equation (2)) leads to conductivities very similar to those obtained with (9).

Desbarats [1992] based his approach on the usefulness and simplicity of the method, but recognized the desirability of theoretical support. Moreover, the fact that a rational number, $1 / 3$, can be found for $\omega$ suggests some theoretical significance.

\subsection{Approach 4: Indelman and Dagan [1993a, b] and Indelman [1993]}

Finally, Indelman and Dagan [1993a, b] considered the general problem of upscaling in anisotropic heterogeneous formations with blocks of any shape. They defined equivalent block conductivity $\widetilde{K}$ as the tensor which maintains energy dissipation in a block. That is, energy dissipated at the block under natural boundary conditions, computed with the $K$ point values from the actual formation, should be equal to the energy computed with $\widetilde{K}$ corresponding to the upscaled formation, under natural boundary conditions. In practice, this equality is pursued only in a statistical sense. Energy dissipation is defined as the rate of dissipation of mechanical energy per unit weight of fluid and given by $e(\mathbf{x})=\nabla h^{t} \cdot \mathbf{K} \cdot \nabla h$. The approach is based on the dissipation energy, a concept that can be considered fundamental, because its minimization leads to the flow equation. In fact, early work on finite element solutions of the flow equation were based on minimizing the numerical approximation to the dissipation function been abandoned in favor of the easier to generalize). Ironically, cretizing the flow domain, so maller than exact dissipation (his would lead to the contradiction of having to use a $\tilde{K}$ larger than the true $K$ for homogeneous fields). Fortunately, in the tests we have performed

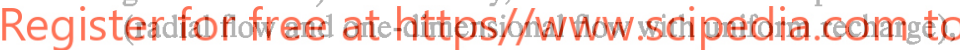
this difference is negligible for any reasonable discretization. In addition to its nice properties (positive-definiteness and additivity), the practical reasons for the choice of $e(x)$ are the following: (1) If the block dissipation computed in the upscaled field, $\tilde{e}(\mathbf{x})$, keeps the statistics (mean and covariance function) of the average dissipation in a block $\bar{e}(\mathbf{x})$, then the global response of the aquifer is identical in the actual and upscaled fields. (2) Flow rates and heads should be very close in the actual and upscaled fields if $\bar{e}(\mathbf{x})$ equals $\tilde{e}(\mathbf{x})$ block to block.

Indelman and Dagan stated a third condition that should be satisfied by $\bar{K}$, namely, that it should be independent of the head gradient. They called this a desirable property and it is much richer in content than it seems on a first glance (for instance, it is what forced us to use the subindex $x x$ in (1)).

The problem with this approach is that it does not provide any formula to compute $\tilde{K}(\mathbf{x})$ for a given block. As a result, comparisons with the other approaches are hard. Therefore we will approach the problem indirectly and show that using a constant conductivity $K_{\text {exx }}$ indeed maintains the average dissipation for the simple boundary conditions defined in (1):

$$
\begin{aligned}
\bar{e}_{\text {exact }} & =-\frac{1}{L_{x} L_{y}} \int_{V} \nabla h \mathbf{q} d V=-\frac{1}{L_{x} L_{y}} \int_{\Gamma} h \mathbf{q} \mathbf{n} d \Gamma \\
& =\frac{Q\left(H_{2}-H_{1}\right)}{L_{x} L_{y}}
\end{aligned}
$$

$$
e\left(K_{e x x}\right)=\overline{\nabla h} K_{e x x} \overline{\nabla h}=\frac{H_{2}-H_{1}}{L_{x}} \frac{Q}{L_{y}}
$$

Therefore according to the third condition of Indelman and Dagan [1993a, p. 919], if a $\widetilde{K}_{x x}$ exists, it should be independent of the local gradient. Thus $\bar{K}_{x x}$ should be equal to $K_{e x x}$, which ensures that the dissipation function is maintained.

Their study is applicable to parallel uniform average flow. They showed that for $\tilde{K}$ to be a scalar random function of isotropic covariance, the block has to be isotropic as well, i.e., a circle in two dimensions. For other block shapes, $\overrightarrow{\mathbf{K}}$ is a tensor of anisotropic covariance. Indelman and Dagan [1993b] and Indelman [1993] derive the first two moments of upscaled permeability for the two most common cases addressed in the literature: two-dimensional isotropic and three-dimensional axisymmetric covariance and for parallelepipedic averaging elements.

\subsection{Summary and Conjectures}

As we have seen, no fewer than four approaches are available for the problem of obtaining equivalent block conductivities. This is somewhat puzzling. It is clear that the hydraulic behavior of a general heterogeneous block cannot be exactly reproduced by a single conductivity, whether scalar or tensor. Hence one is forced to define block conductivity in some approximate way. Depending on the criteria used in the defini-

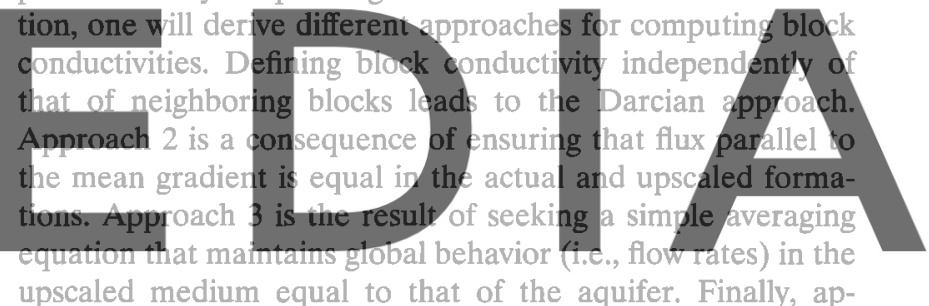

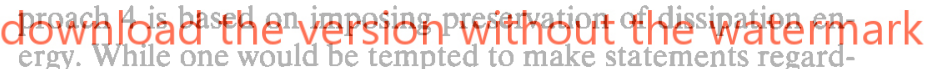
ing the relative merits of the approaches, the discussion presented thus far suggests that they are indeed very similar.

We find it puzzling that this apparent similarity has not been reflected in the literature, thus far. In fact, some of the published findings are rather disturbing. For example, Indelman [1993] concluded that the expected value of conductivity for square blocks is anisotropic, even for isotropic covariances. No theoretical support exists for the appealing approach by Desbarats. Indelman and Dagan [1993] stated that the effective conductivity derived from the upscaling of Rubin and GómezHernández [1990] is different from that derived from point values. Rubin and Gómez-Hernández [1990] concluded that $\left\langle K_{b}\right\rangle$ decreases with block size. Some of these apparent puzzles may be caused by the fact that Rubin and Gómez-Hernández worked with $K_{b}$, instead of $Y_{b}=\ln K_{b}$. Hence our first task will be to derive the statistics of $Y_{b}$ using the definition of Rubin and Gómez-Hernández. This choice is not based on any assumption about the superiority of this method, but rather on the fact that it is the most manageable. In any case, such an approach will suffice to solve the above puzzles, as shown in the next two sections. The rest of the paper will be devoted to verifying the conjecture about the similarity of all approaches. This will be achieved numerically for the most part, but we will prove analytically that working with $K_{b}$, as defined by Rubin and Gómez-Hernández [1990], indeed keeps the expected value and covariance function of the average dissipation function. 


\section{Proposed Methodology and Statistics of $Y_{b}$}

\subsection{Analytical Developments}

This section is devoted to deriving the statistics of $Y_{b}$ based on the definition by Rubin and Gómez-Hernández [1990] (section 2.3). The starting point will be (3) which can be written in a shorter form:

$$
\overline{q_{\imath}(\mathbf{x})}=-K_{b}(\mathbf{x}) \overline{j_{t}(\mathbf{x})} \quad i=1, \cdots, n
$$

where the overbar stands for volumetric average, $\mathbf{j}(\mathbf{x})$ is our notation for head gradient, $n$ is dimensionality of the problem, and $\mathbf{q}(\mathbf{x})$ represents flow at the local scale, which is given by Darcy's law:

$$
\mathbf{q}(\mathbf{x})=-K(\mathbf{x}) \mathbf{j}(\mathbf{x})
$$

where $K(\mathbf{x})$ is point hydraulic conductivity value, taken as a scalar.

Considering mean gradient uniform and parallel to the $x$ direction and expanding both $\mathbf{q}(\mathbf{x})$ and $\mathbf{j}(\mathbf{x})$ in their expected value plus a perturbation (in a classical small-perturbation approach; e.g., see Dagan [1989]), we get

$$
\left\langle q_{i}\right\rangle+\overline{q_{i}^{\prime}(\mathbf{x})}=-K_{b}(\mathbf{x})\left[\left\langle j_{l}\right\rangle+\overline{j_{i}^{\prime}(\mathbf{x})}\right]
$$

where $\left\langle q_{1}\right\rangle$ and $\left\langle j_{1}\right\rangle$ (which do not depend on $\mathbf{x}$ for the uniform mean flow case) stand for expected values of $q(x)$ and $j(x)$

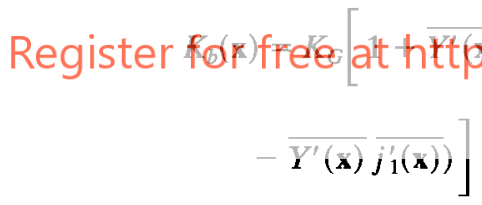

Now, taking logarithms,

$$
\begin{aligned}
Y_{b}(\mathbf{x}) & =\ln K_{G}+\ln \left[1+\overline{Y^{\prime}(\mathbf{x})}+\frac{1}{2} \overline{Y^{\prime 2}(\mathbf{x})}\right. \\
& +\frac{1}{\left\langle j_{1}\right\rangle}\left(\overline{Y^{\prime}(\mathbf{x}) j_{1}^{\prime}(\mathbf{x})}-\overline{Y^{\prime}(\mathbf{x})} \overline{\left.j_{1}^{\prime}(\mathbf{x})\right)}\right]
\end{aligned}
$$

and assuming again small perturbations, we can write

$$
\begin{aligned}
Y_{b}(\mathbf{x}) & =\ln K_{G}+\overline{Y^{\prime}(\mathbf{x})}+\frac{1}{2} \overline{Y^{\prime 2}(\mathbf{x})}+\frac{1}{\left\langle j_{1}\right\rangle}\left(\overline{Y^{\prime}(\mathbf{x}) j_{1}^{\prime}(\mathbf{x})}\right. \\
& \left.-\overline{Y^{\prime}(\mathbf{x})} \overline{j_{1}^{\prime}(\mathbf{x})}\right)-\frac{1}{2}\left(\overline{Y^{\prime}(\mathbf{x})}\right)^{2}+O\left(\sigma_{Y}^{3}\right)
\end{aligned}
$$

Treating $Y_{b}(\mathbf{x})$ as an ergodic random function, we can derive its first two statistical moments. For its expected value (which again does not depend on the location $\mathbf{x}$ ), and taking into account that volumetric average and expected value (averaging over the ensemble of realizations) are interchangeable, we get, after dropping the terms higher than second order in $\sigma_{Y}$

$$
\left\langle Y_{b}\right\rangle=\langle Y\rangle+\frac{1}{2} \sigma_{Y}^{2}+\frac{1}{\left\langle j_{1}\right\rangle}\left(C_{Y_{j_{1}}}(0)-\overline{C_{Y_{1}}}\right)-\frac{1}{2} \overline{C_{Y}}
$$

where $\overline{C_{Y}}$ and $\overline{C_{Y_{J_{1}}}}$ are short forms of the averages of the covariance functions throughout the block $V$. These short forms are given by

$$
\begin{gathered}
\overline{C_{Y}}=\frac{1}{V^{2}} \int_{V} \int_{V} C_{Y}\left(\mathbf{x}, \mathbf{x}^{\prime}\right) d \mathbf{x} d \mathbf{x}^{\prime} \\
\overline{C_{Y_{11}}}=\frac{1}{V^{2}} \int_{V} \int_{V} C_{Y_{j 1}}\left(\mathbf{x}, \mathbf{x}^{\prime}\right) d \mathbf{x} d \mathbf{x}^{\prime}
\end{gathered}
$$

$C_{Y j_{1}}\left(\mathbf{x}, \mathbf{x}^{\prime}\right)$ is the cross covariance $\left(C_{Y_{j_{1}}}(0)=C_{Y_{J_{1}}}(\mathbf{x}, \mathbf{x})\right.$ being the cross variance) between $Y(\mathbf{x})$ and $\partial / \partial x^{\prime}\left[h\left(\mathbf{x}^{\prime}\right)\right]$. This can be expressed as a function of the cross covariance between $Y(\mathbf{x})$ and $h\left(\mathbf{x}^{\prime}\right)$ (that is, $C_{Y h}\left(\mathbf{x}, \mathbf{x}^{\prime}\right)$ ) by

$$
C_{Y_{1}}\left(\mathbf{x}, \mathbf{x}^{\prime}\right)=\left\langle Y^{\prime}(\mathbf{x}) j_{1}^{\prime}\left(\mathbf{x}^{\prime}\right)\right\rangle=\frac{\partial}{\partial x^{\prime}} C_{Y h}\left(\mathbf{x}, \mathbf{x}^{\prime}\right)
$$

and as $C_{Y_{J_{1}}}(0)=-\left(\sigma_{Y}^{2} / n\right)\left\langle j_{1}\right\rangle$ for the case of statistical isotropy [e.g., Gutjahr et al., 1978], (21) can be rewritten

$$
\left\langle Y_{b}\right\rangle=\langle Y\rangle+\left(\frac{1}{2}-\frac{1}{n}\right) \sigma_{Y}^{2}-\frac{1}{\left\langle j_{1}\right\rangle} \overline{C_{Y_{11}}}-\frac{1}{2} \overline{C_{Y}}
$$

This formula is independent of block shape. Later, we will find a simplified result for the particular case of squared or cubic blocks. Now we want to derive the corresponding second-order moments; for that purpose we first obtain the pertufbation
\[ \mathbf{Y}_{b}^{\prime}(\mathbf{x})=\overline{Y^{\prime}}(\mathbf{x})+O\left(\boldsymbol{\sigma}_{Y}^{2}\right) \]
From here we can find the covgriance function, which is given
by download the version without the watermark

$$
=\frac{1}{V^{2}} \int_{V(\mathbf{x})} \int_{V\left(\mathbf{x}^{\prime}\right)} C_{Y}\left(\mathbf{x}, \mathbf{x}^{\prime}\right) d \mathbf{x} d \mathbf{x}^{\prime}+O\left(\sigma_{Y}^{3}\right)
$$

where $V(\mathbf{x})$ is the symbol for a volume $V$ centered at point $\mathbf{x}$. From this expression we can directly find the variance by just making $V(\mathbf{x})=V\left(x^{\prime}\right)$ and thus getting, after dropping the terms higher than second order in $\sigma_{Y}$,

$$
\sigma_{Y_{b}}^{2}=\overline{C_{Y}}
$$

The variance of $Y_{b}(\mathbf{x})$ with respect to block size is plotted in Figure 2 for the three most common variogram models (exponential, spherical, and Gaussian) for a two-dimensional isotropic case and squared upscaling blocks. In this and the following figures, block size is normalized by the integral scale $I$. Integration of the covariance function corresponding to (22) was carried out numerically using a Gauss-Cauchy algorithm with 16 Gauss points per square. It can be seen that in plotting $\sigma_{Y_{b}}^{2}$ (normalized by $\sigma_{Y}^{2}$ ) versus block size, the three variogram models give very similar curves (particularly for large block sizes). Equation (28) can also be written as

$$
\sigma_{Y_{b}}^{2}=\alpha \sigma_{Y}^{2}
$$

where $\alpha=\overline{C_{Y}} / \sigma_{Y}^{2}$ is a function of normalized block size and variogram model selected, as shown in Figure 2.

The limiting cases can be easily obtained. For very small 


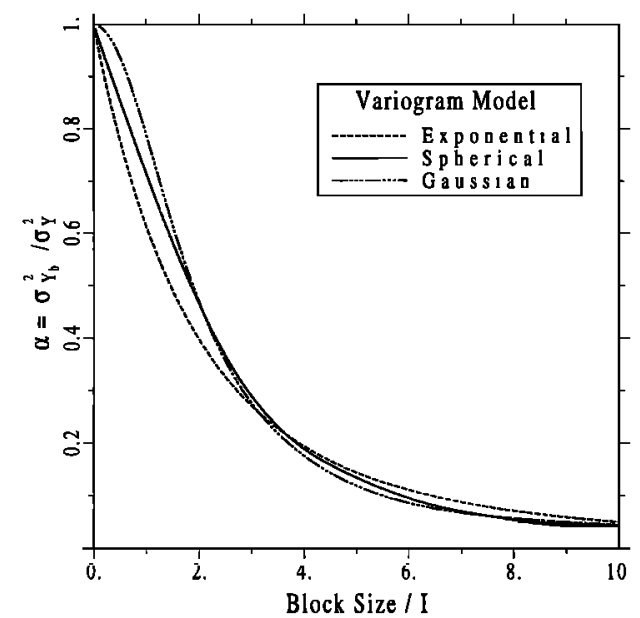

Figure 2. Normalized variance of $Y_{b}\left(\sigma_{Y_{b}}^{2} / \sigma_{Y}^{2}\right)$ versus normalized block size for three different variogram models of $Y$ in a two-dimensional domain.

blocks we have $V \rightarrow 0, \overline{C_{Y}}=\sigma_{Y}^{2}$ and $\overline{C_{Y j_{1}}}=-\left(\sigma_{Y}^{2} / n\right)\left\langle j_{1}\right\rangle$, so that

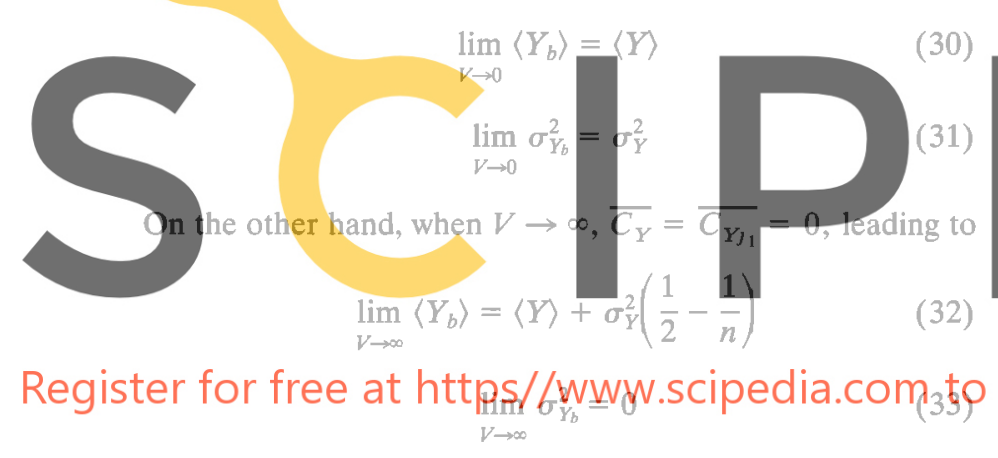

As a result, we get that for small blocks (at the point scale), block and point values coincide (as should be the case). For very large blocks, (32) and (33) imply that $Y_{b}$ tends to a single deterministic value (the variance tends to zero) so that

$$
\lim _{V \rightarrow \infty} K_{b}=\exp \left(\lim _{V \rightarrow \infty}\left\langle Y_{b}\right\rangle\right)=K_{G} \exp \left[\sigma_{Y}^{2}\left(\frac{1}{2}-\frac{1}{n}\right)\right]
$$

which is exactly the value for the effective hydraulic conductivity derived by Gutjahr et al. [1978], among others, for statistically isotropic media, written in an exponential form (as suggested by Gelhar and Axness [1983]). In short, these results validate (25) and (28), at least for the limiting cases.

The next step is to go back to (25) and develop a simplified expression for the simple shapes analyzed in this work (square and cubes). For this purpose we need to relate $\overline{C_{Y_{1}}}$ to $\overline{C_{Y}}$. This is carried out in Appendix $A$, where it is found that for square $(n=2)$ or cubic $(n=3)$ blocks

$$
\overline{C_{Y_{j 1}}}=-\frac{\left\langle j_{1}\right\rangle}{n} \overline{C_{Y}}
$$

independent of block size (naturally, block size is already included in the expression of $\overline{C_{Y}}$ ). When substituting this expression into (25), we arrive at the following expression

$$
\left\langle Y_{b}\right\rangle=\langle Y\rangle+\sigma_{Y}^{2}\left(\frac{1}{2}-\frac{1}{n}\right)-\overline{C_{Y}}\left(\frac{1}{2}-\frac{1}{n}\right)
$$

and writing again $\overline{C_{Y}}$ as $\alpha \sigma_{Y}^{2}((28)$ and (29)), we get a very simple formula:

$$
\left\langle Y_{b}\right\rangle=\langle Y\rangle+\left(\frac{1}{2}-\frac{1}{n}\right)(1-\alpha) \sigma_{Y}^{2}
$$

Equations (28) and (37) are the final expressions for the two first statistical moments of $Y_{b}$ for square $(n=2)$ or cubic $(n=3)$ blocks, valid up to first order in $\sigma_{Y}^{2}$. Expressions identical to (28) and (37) could also be obtained separately from (18) by assuming that $Y_{b}$ is a multi-Gaussian random function. In this case we can relate the mean and variance of $Y_{b}$ with the statistical moments of $K_{b}$ (which were given by Gómez-Hernández [1991]) using the following expressions:

$$
\begin{gathered}
\left\langle Y_{b}\right\rangle=\ln \left\langle K_{b}\right\rangle-\left(\sigma_{Y_{b}}^{2} / 2\right) \\
\sigma_{Y_{b}}^{2}=\ln \left[1+\left(\sigma_{K_{b}}^{2} /\left\langle K_{b}\right\rangle^{2}\right)\right]
\end{gathered}
$$

and dropping again the terms higher than first order in $\sigma_{Y}^{2}$. This assumption of multi-Gaussianity of $Y_{b}$ is based on the work by Fenton and Griffiths [1993], who used a Monte Carlo approach to conclude that the probabilistic distribution of block conductivities could be considered lognormal up to, at least, $\sigma_{Y}^{2}=2.83$. The advantage of the derivation adopted in the paper (equations (20) and on) with respect to the one
suggested in the last paragrapk is that we avoid the nced for
assumptions about the distribution of $Y_{b}$.
3.2. Discussion of the Two-Dimensional Case
We start by analyzing the statistical moments of $Y_{b}$ devel-
oped in the previous section for a general case, apply them to the two-dimensional case, and move later to the threedimensional case. Substituting $n=2$ into (37), we get download the version without the watermark

so up to first order in $\sigma_{\mathrm{Y}}^{2}$, we obtain

$$
\exp \left(\left\langle Y_{b}\right\rangle\right)=K_{G}
$$

That is, the expected value of $Y_{b}$ remains constant (up to first order in $\sigma_{Y}^{2}$ ) and equal to $\ln K_{G}$, for any size of the averaging square block. On the other hand, the variance decreases, going from the original point value, $\sigma_{Y}^{2}$, to zero, as the block size increases (equations (29), (31), and (33)). At the limit, for very large block sizes, we get a Dirac delta distribution for the random variable $Y_{b}$, i.e., a deterministic value, $Y_{b}=\ln K_{G}$ (which, in this case, coincides with $\ln K_{\text {eff }}$ ). On the contrary, $\left\langle K_{b}\right\rangle$ is not invariant with block size; for very large blocks, $\left\langle K_{b}\right\rangle$ tends to $K_{G}$ (thus equal to $K_{\text {eff }}$ ), as the decrease in the variance makes the lognormal and normal distributions very similar. Instead, for small values, $\left\langle K_{b}\right\rangle$ tends to the arithmetic mean of the point $K$ values. This explains the decrease in $\left\langle K_{b}\right\rangle$ versus block size observed by Gómez-Hernández [1991].

If we now consider $Y_{b}$ to be multi-Gaussian, as suggested in the previous section, we can derive the upscaled effective conductivity $\tilde{K}_{\text {eff }}$ in a two-dimensional domain as equal to exp $\left(\left\langle Y_{b}\right\rangle\right)=K_{G}$ (this is based on Matheron [1967], among many others), thus verifying one of the conditions stated by Indelman and Dagan [1993a], namely, that the effective value in the upscaled medium must preserve that in the actual formation, $K_{\text {eff }}$. We must state that this condition is not satisfied if $K_{b}$ is 


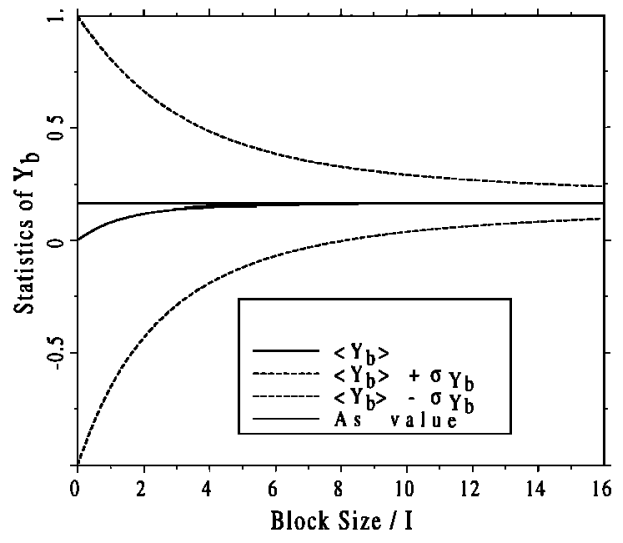

Figure 3. Statistical moments (mean and plus or minus one standard deviation) of $Y_{b}$ as a function of normalized block size, for the three-dimensional isotropic case. Variogram model for $Y$ is exponential. The asymntotic value of $K$, for large blocks is also displayed.

considered to be multi-Gaussian. Later, we will check the remaining conditions stated by Indelman and Dagan, for a particular numerical case.

Our solution compares exactly with that of Desbarats [1992]. Substituting $\omega=0$ (corresponding to two dimensions) in (10) and (11), we get (29) and (40).

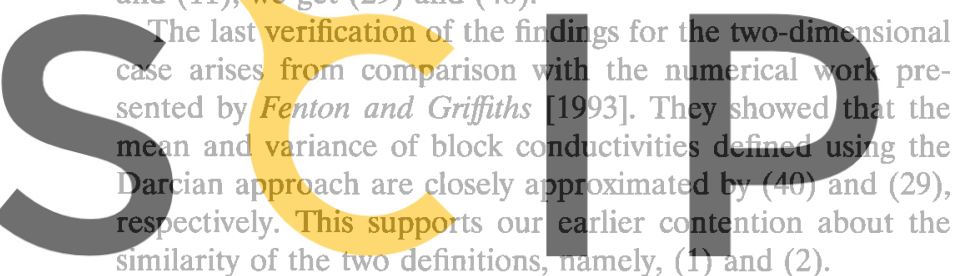

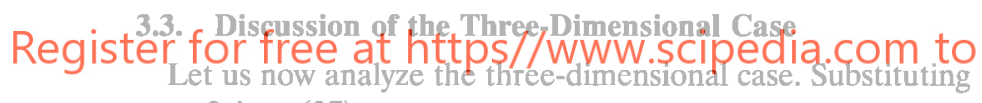
$n=3$ into (37), we get

$$
\left\langle Y_{b}\right\rangle=\langle Y\rangle+\frac{1}{6}(1-\alpha) \sigma_{Y}^{2}
$$

so that, up to first order in $\sigma_{Y}^{2}$, we obtain

$$
\exp \left(\left\langle Y_{b}\right\rangle\right)=K_{G} \exp \left[\frac{1}{6}(1-\alpha) \sigma_{Y}^{2}\right]
$$

In this case it is found that $\left\langle Y_{b}\right\rangle$ grows from the starting value in the original field, $\langle Y\rangle$, to the effective value derived in the literature (as $\alpha=1$ for small blocks and $\alpha=0$ for large ones). To check our formulation, we can compute $\tilde{K}_{\text {eft }}$ again, as we did for the two-dimensional case. By assuming statistical isotropy of $Y_{b}$, we can again use here the formula developed by Gutjahr et al. [1978] for the three-dimensional case written in exponential form:

$$
\widetilde{K}_{\text {eff }}=\exp \left(\left\langle Y_{b}\right\rangle+\frac{1}{6} \sigma_{Y_{b}}^{2}\right)
$$

By substituting (42) and (29) in (44) we get

$$
\begin{aligned}
\bar{K}_{\mathrm{eff}} & =\exp \left(\langle Y\rangle+\frac{1}{6}(1-\alpha) \sigma_{Y}^{2}+\frac{1}{6} \alpha \sigma_{Y}^{2}\right) \\
& =\exp \left(\langle Y\rangle+\frac{1}{6} \sigma_{Y}^{2}\right)=K_{\mathrm{eff}}
\end{aligned}
$$

so that the necessary condition stated by Indelman and Dagan [1993a] is again verified. Figure 3 shows the statistics of $Y_{b}$ as a function of block size for $\sigma_{Y}^{2}=1.0$ and $\langle Y\rangle=0$. In the figure, we plot the expected value $\left\langle Y_{b}\right\rangle$ (equation (42)), as well as plus or minus one standard deviation ( $\pm \sigma_{Y_{b}}$, computed from (29)). It is clear from the figure that for very large blocks $K_{b}$ tends toward a single deterministic value (as the variance decreases with block size), which corresponds exactly to the effective hydraulic conductivity in a three-dimensional infinite domain. This result can only be compared in the analytical literature to that given by Indelman [1993], since neither Rubin and Gómez-Hernández [1990] nor Gómez-Hernández [1991] addressed the three-dimensional case. Indelman considered that the expected value of block conductivity, $\langle\overline{\mathbf{K}}\rangle$, is an anisotropic tensor, even for blocks of cubic shape in isotropic domains, with the only exception for the limiting case of small blocks. Otherwise, the horizontal component, $\left\langle\widetilde{K}_{11}\right\rangle$, is always larger than the vertical one, $\left\langle\widetilde{K}_{33}\right\rangle$. In our methodology, $\left\langle Y_{b}\right\rangle$ is taken as a scalar, independent of the mean flow direction, and is given by (42). Figure 4 is a comparison between the two formulations. In the figure we plot the first-order expansion of exp $\left(\left\langle Y_{b}\right\rangle\right) / K_{G}$ (which is equal to $1+\frac{1}{6}(1-\alpha) \sigma_{Y}^{2}$ from (43)), for the particular case $\sigma_{Y}^{2}=0.5$. These values were the same as used by Indelman to obtain results for the three-dimensional isotropic case with cubic upscaling blocks [Indelman, 1993, Figure 8] and thus allow direct comparison. In Figure $4, K_{G 1}$ and $K_{G 3}$ indicate the geometric means of the tensorial components in the horizontal $\left(K_{G 1}=K_{G 2}\right)$ and vertical $\left(K_{G 3}\right)$ directions, given by Indelman. $K_{\text {eff }}$ is shown for purposes of comparison. It is possible to derive analytically that up to first

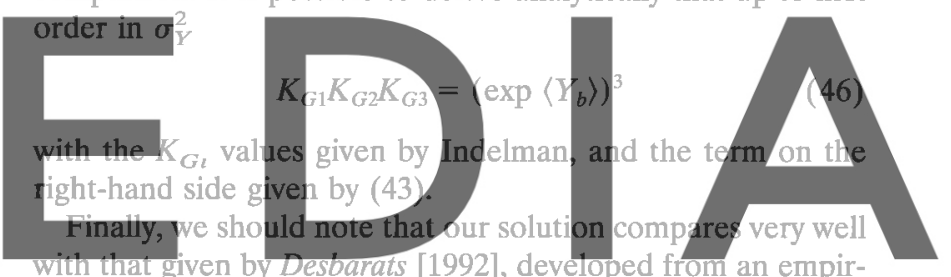
with that given by Desbarats [1992], devetoped from an empirical averaging rule (equation (9)). By using a value of $\omega=1 / 3$

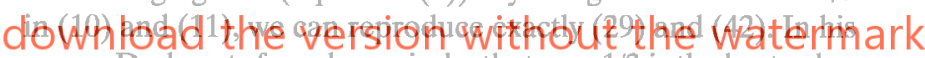
paper, Desbarats found, precisely, that $\omega=1 / 3$ is the best value that fits his numerical simulations. This can be taken as a numerical proof of our methodology for the three-dimensional case. Furthermore, Desbarats stated that the fact that power $\omega$ turned out to be a rational number suggested some theoretical significance. Our findings indeed support this contention.

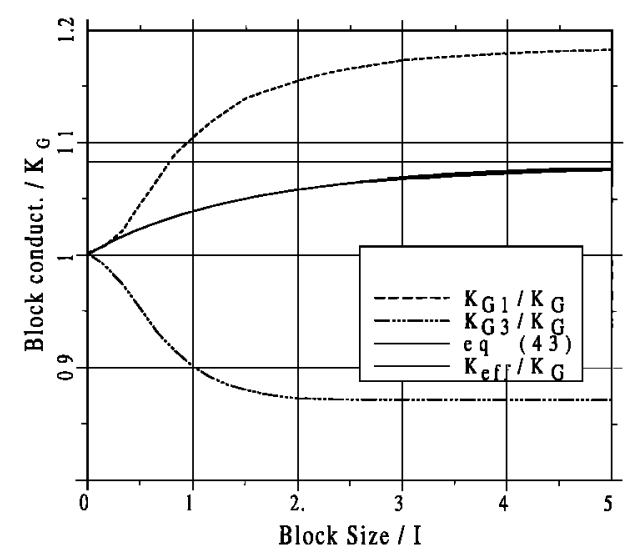

Figure 4. Comparison between directional geometric means of block conductivity values [Indelman, 1993] with a scalar approach (equation (43)), for $\sigma_{Y}^{2}=0.5$. Values are presented versus normalized block size. 


\section{Numerical Simulations: Check on Analytical Developments}

This section is devoted to numerical simulations in twodimensions, intended to confirm (or reject) the analytical results derived in section 3 . In the next section we will use the same simulations to compare the different formulations in terms of total energy dissipation. This will allow us to check our basic conjecture, the similarity of all methodologies. At this point our general idea is to generate a two-dimensional heterogeneous hydraulic conductivity field and to use the methodology of upscaling with different block sizes to find the sample mean and variance of $Y_{b}$. These numbers are compared with the analytical developments in section 3 and found to be in excellent agreement.

The first step consisted of generating a two-dimensional field. We considered a square domain, discretized into $420 \times$ 420 square elements of equal size. Each of them was assigned a $K$ value (values defined over this small support will be considered as "point values"). The 176,400 values were generated with the program GCOSIM3D [Gómez-Hernández and Journel, 1993], which uses a sequential simulation algorithm to generate realizations from a multi-Gaussian random function. For that reason the hydraulic conductivity field was taken as one of all the possible realizations from a multi-Gaussian random function $Y$, with constant mean and isotropic, stationary correlation structure. We used a spherical correlation function, with an integral scale equal to 5 units of length. The statistics of the generated field are sample sample variance, $s_{Y}^{2}=1.005 . S$ point $Y$ values was checked by efficient $(\mathrm{AC})$, equal to -0.04 that the input conditions were sa (Figure 5) and the raw variograms in the $x$ and $y$ directions (Figure 6). This allows us to check both Gaussianity of the point values and isotropy in the correlation structure. Despite

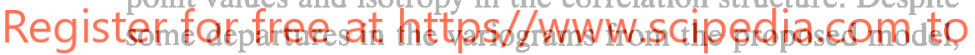
we can consider the realization to be representative of the input conditions. Figure 7 is a grey plot of the final field; it is a four-tone plot, where point $K$ values, classified in quartiles, range from solid (high values) to open (low ones).

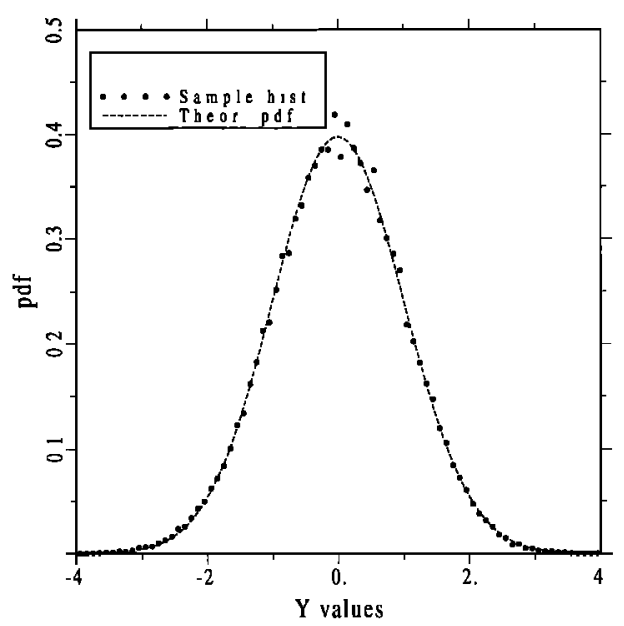

Figure 5. Probability density function (pdf) corresponding to the final $Y$ field generated with GCOSIM3D. The pdf corresponding to a Gaussian distribution with the same mean and variance is plotted for purposes of comparison.

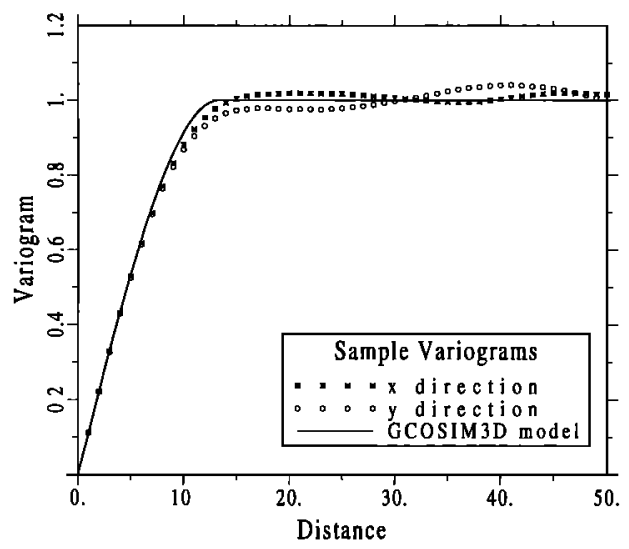

Figure 6. Exhaustive sample variograms in the $x$ and $y$ directions for the final $Y$ field. Model used as input in GCOSIM3D is also shown for purposes of comparison.

The second step involved solving flow in this field for some simple predefined boundary conditions. We imposed a constant mean gradient in the $x$ direction. This is easily done in a rectangular domain aligned with the coordinate axis, by imposing no-flow conditions on the sides parallel to the $x$ axis and constant, but different, prescribed head values $\left(\mathrm{H}_{1}, \mathrm{H}_{2}\right)$ on the sides parallel to the $y$ axis. Then, the mean gradient $\left\langle j_{1}\right\rangle$ equals $\left(\mathrm{H}_{2}-H_{1}\right) / L_{x}, L_{x}$ being the total length in the $x$ direction.
The flow solver used was a firite element computer code,
FAITH [Sanchez-Vila et al., 1993], which is specially defigned
to solve the steady state flow and transient solute transport
equations in a two-dimensional heterogeneous nedium dis-
cretized into a very large number of rectangular elements (up
to a few humdred thousand).

The final step consisted of computing the $K_{b}(\mathrm{x})$ values. We

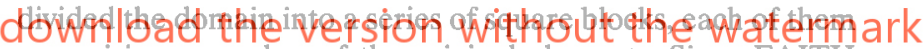
comprising a number of the original elements. Since FAITH gives, as an optional output, the head value at each node and/or the head gradient at each element, the integrais in (2) can be approximated by sums over the elements that form a block. Hence $K_{b}$ is given by

$$
K_{b}(\mathbf{x})=\frac{\sum_{e} K_{e} \frac{\partial}{\partial x} h_{e}}{\sum_{e} \frac{\partial}{\partial x} h_{e}}
$$

where $e$ stands for values computed at the element and the sum extends to all elements included in a block $V$.

The same process was repeated for different block sizes. Statistics of the $K_{b}$ values thus computed are shown in Table 1, which contains the sample mean $\left(Y_{b, m}\right)$, sample variance $\left(s_{Y_{b}}^{2}\right)$ and asymmetry coefficient (AC) of $Y_{b}$ for different block sizes. Also shown in Table 1 is the factor $F_{b, m}$, defined as

$$
F_{b, m}=\exp \left(Y_{b, m}\right) / K_{G}
$$

Equation (41) indicates that under ergodicity, this factor should remain equal to one for any block size in a twodimensional case. For blocks larger than $84 \times 84$ elements, the second- and third-order moments were not computed, because the number of $Y_{b}$ values was not enough to obtain meaningful statistics. In order to compare sample variances $\left(s_{Y_{b}}^{2}\right)$ with 


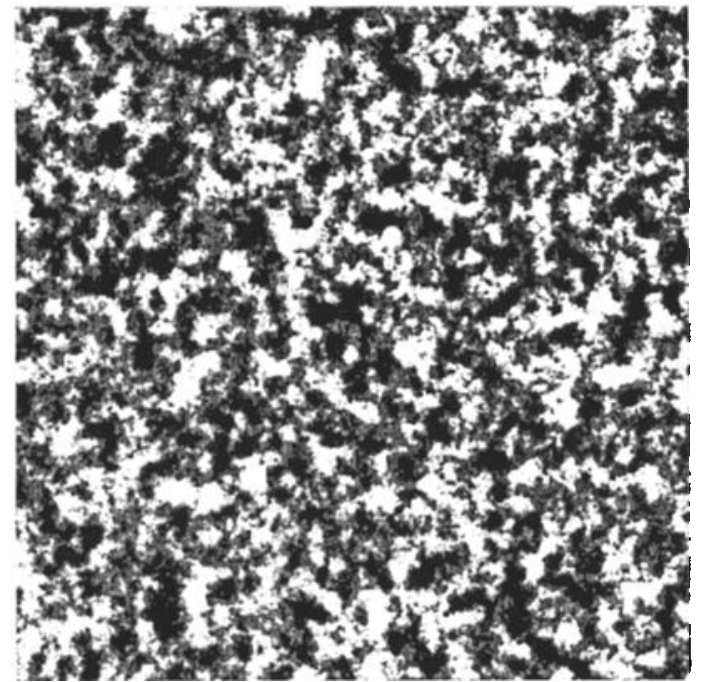

Figure 7. Final $K$ field with point values classified into quartiles. The more heavily shaded areas denote higher point $K$ values.

theoretical values $\left(\sigma_{Y_{b}}^{2}\right)$, Table 1 also displays what we note as corrected variances $\left(s_{Y_{b_{f}}}^{2}\right)$. This correction factor accounts for the difference between "real point values" and "point values" at the element size used for numerical computations. Theoretical statistical moments were defived by assuming a tocal scale which is very small compared numerical simulation we considar local scale to be one fifth of the in to the smallest possible blocks (1 cing out the variability at small the integr
egral scale.
1 elements
ler scales. A
13 for the ming a local scale
tical reasons, the
Thus when going
s), we are already
As the theoretical
spherical variogram, the sample variances are corrected by this value.

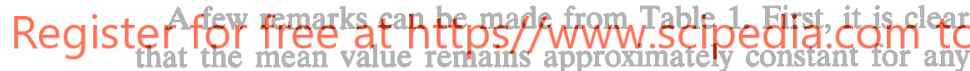
block size, even though the small-perturbation hypothesis $\left(\sigma_{Y}^{2} \ll 1\right)$ is not met. In fact, the values of $F_{b, m}$ never differ by more than $0.8 \%$ from the theoretical value 1.0 , in agreement with (41). The small differences can be attributed to the finite size of the domain and to the nonperfect fit between the model and the two directional variograms. A small departure of the variograms could account for a slight anisotropy, which can cause the mean to deviate from its theoretical value. The fit would be much better if we averaged a number of different realizations (Monte Carlo), instead of a single one. In any case, the agreement can be considered excellent.

We can now compare the analytical expression for the variance of $Y_{b}$, which is given by (29), with the corrected sample variances in Table 1. This is done in Figure 8, where we plot theoretical $\left(\sigma_{Y_{b}}^{2}\right.$, equation (29)) versus corrected sample variances $\left(s_{Y_{b, c}}^{2}\right)$. Although, again, (29) was obtained by considering $\sigma_{Y}^{2}$ much smaller than one (here it is 1.005 ), we can see that the results tend to agree very well.

The third important issue to analyze is Gaussianity of the block conductivity univariate distribution. We can check this assumption in our numerical example by looking at $\mathrm{AC}$ values in Table 1. In a symmetrical distribution (such as Gaussian) this value should remain equal to zero. We can see that $\mathrm{AC}$ always remains small, except for blocks bigger than $35 \times 35$ elements, possibly due to the reduction in the sample size. Anyway, a visual confirmation of both symmetry and Gaussi- anity can be obtained by the representation of the normalized histogram. This is done in Figure 9 for a couple of block sizes, $10 \times 10$ and $20 \times 20$ (corresponding to 2 and 4 times the integral scale). In the same plot the theoretical probability density functions (pdf) of two Gaussian distributions with the same means and variances (taken from Table 1) are displayed. Agreement is considered very good.

\section{Numerical Simulations: Check on Necessary and Sufficient Conditions}

As discussed earlier, Indelman and Dagan [1993a] state that any upscaling methodology should satisfy a number of conditions. This section is devoted to analyzing those conditions for the different methodologies considered throughout the paper.

The first step consisted of generating block conductivity values. We considered two methodologies: Darcian (section 2.1) and Rubin and Gómez-Hernández (summarized in section 2.2). Desbarats's methodology is not analyzed here, as it was already studied in his paper [Desbarats, 1992] and found to be very similar to both approaches, at least up to $\sigma_{Y}^{2}=1.0$. We consider again our original heterogeneous field (shown in Figure 7) and select a certain block size, $10 \times 10$ elements (two integral scales). The $K_{b}$ values for the second methodology were already found in the previous section (the statistics appear in Table 1). For the Darcian methodology we proceeded as follows: We selected blocks of size $10 \times 10$, isolated them. from the rest of the medium, and solved the flow problem for
each single block under the conditions stated in section 2.1 .
From here we could obtain the $K_{\text {ext }}$ conductivity values. Figure
10 shows the correlation between the values obtained by the
two approaches; the $1: 1$ line is aiso plotted. Correlation can be
considered excellent. We shoyld note again tha $\sigma_{Y}^{2}=1.0$,
and that the correlation will not necessarily be as good for higher variances.

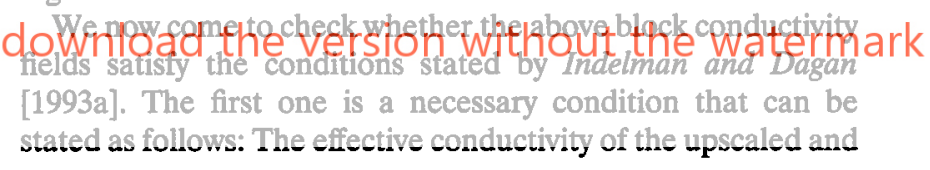

Table 1. Sample Statistics of $Y_{b}$ for Different Block Sizes

\begin{tabular}{|c|c|c|c|c|c|c|}
\hline $\begin{array}{c}\text { Block } \\
\text { Size }\end{array}$ & $Y_{b, m}$ & $F_{b, m}$ & $s_{Y_{b}}^{2}$ & $s_{Y_{b, c}}^{2}$ & $\sigma_{Y_{b}}^{2}$ & $\mathrm{AC}$ \\
\hline $1 \times 1$ & -0.0012 & 1.000 & 1.005 & 0.946 & 0.946 & -0.041 \\
\hline $2 \times 2$ & 0.0053 & 1.000 & 0.910 & 0.856 & 0.887 & -0.049 \\
\hline $3 \times 3$ & 0.0063 & 1.006 & 0.834 & 0.785 & 0.830 & -0.054 \\
\hline $4 \times 4$ & 0.0061 & 1.007 & 0.783 & 0.738 & 0.772 & -0.059 \\
\hline $5 \times 5$ & 0.0065 & 1.008 & 0.728 & 0.685 & 0.717 & -0.061 \\
\hline $6 \times 6$ & 0.0063 & 1.007 & 0.676 & 0.637 & 0.662 & -0.067 \\
\hline $7 \times 7$ & 0.0065 & 1.008 & 0.618 & 0.582 & 0.610 & -0.060 \\
\hline $10 \times 10$ & 0.0066 & 1.008 & 0.481 & 0.453 & 0.467 & -0.117 \\
\hline $12 \times 12$ & 0.0067 & 1.008 & 0.412 & 0.388 & 0.386 & -0.179 \\
\hline $15 \times 15$ & 0.0060 & 1.007 & 0.314 & 0.295 & 0.291 & -0.111 \\
\hline $20 \times 20$ & 0.0040 & 1.005 & 0.219 & 0.206 & 0.190 & -0.112 \\
\hline $28 \times 28$ & 0.0048 & 1.006 & 0.136 & 0.128 & 0.110 & -0.106 \\
\hline $30 \times 30$ & 0.0039 & 1.005 & 0.106 & 0.099 & 0.096 & -0.034 \\
\hline $35 \times 35$ & 0.0001 & 1.001 & 0.082 & 0.078 & 0.071 & -0.240 \\
\hline $42 \times 42$ & 0.0023 & 1.003 & 0.060 & 0.057 & 0.050 & -0.279 \\
\hline $60 \times 60$ & 0.0035 & 1.004 & 0.035 & 0.033 & 0.035 & -0.146 \\
\hline $84 \times 84$ & 0.0032 & 1.004 & 0.019 & 0.018 & 0.022 & -0.259 \\
\hline $105 \times 105$ & 0.0043 & 1.005 & $\cdots$ & $\ldots$ & $\ldots$ & ... \\
\hline $140 \times 140$ & 0.0037 & 1.005 & $\ldots$ & $\ldots$ & $\ldots$ & $\ldots$ \\
\hline $210 \times 210$ & 0.0036 & 1.005 & $\cdots$ & $\ldots$ & $\ldots$ & $\ldots$ \\
\hline $420 \times 420$ & 0.0045 & 1.006 & $\ldots$ & $\ldots$ & $\ldots$ & ... \\
\hline
\end{tabular}




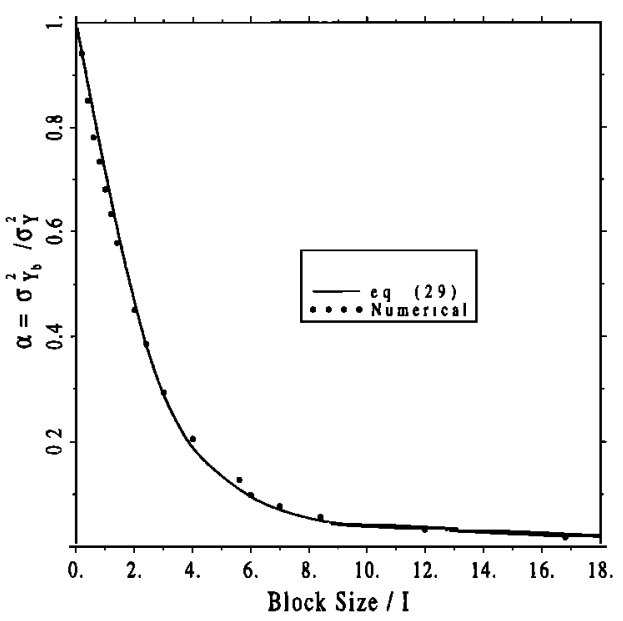

Figure 8. Comparison between $Y_{b}$ variances; corrected $\left(s_{Y_{b, c}}^{2}\right.$ in Table 1) and theoretical $\left(\sigma_{Y_{b}}^{2}\right.$ in $\left.(29)\right)$.

actual formations must be equal. This was analyzed for the approach by Rubin and Gómez-Hernández in section 3. The last condition, considered as "desirable" by the authors, is that the block conductivity should depend only on the statistical structure and on block partitioning and not on head gradient. It is easy to see that this is verified by the Darcian approach. As the two methodologies give very similar values (Figure 10), we conclude that indeed both of them provide good agreement of the two conditions previously stated. In any case, the conditions we want now to focus on are the "sufficient" ones, which can be expressed in terms of the dissipation function, defined in section 2.4. "Sufficient" conditions mean that energy dissipation in a block should be equal for both the actual medium, $\bar{e}(\mathbf{x})$, and the upscaled medium, $\tilde{e}(\mathbf{x})$. In reality, since both $\bar{e}$ and $\tilde{e}$ are random functions, these conditions can only be pursued in a statistical sense. Then, equality of the first two moments of the random functions becomes a sufficient condition for the necessary one.

For purposes of comparison we first have to compute the "true" (reference) dissipation values. We considered again our blocks of size $2 I \times 2 I$. As we already solved the flow problem

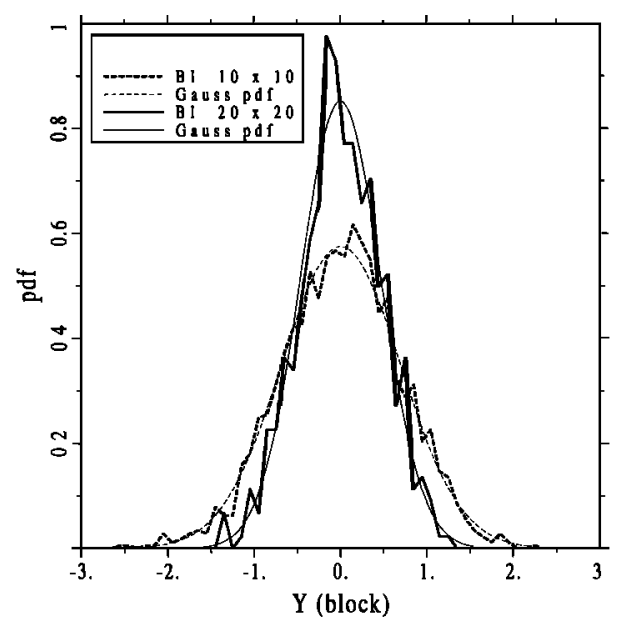

Figure 9. Sample-normalized histogram of $Y_{b}$ for $10 \times 10$ and $20 \times 20$ block sizes. The pdfs corresponding to Gaussian distributions with the same means and variances are also shown.

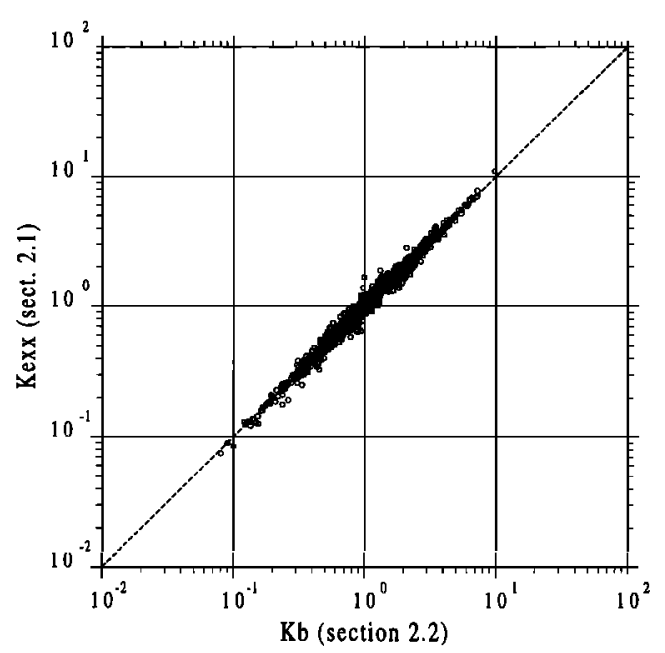

Figure 10. Comparison between block conductivity values given by the Darcian $\left(K_{e x x}\right)$ and Rubin and Gómez-Hernández's $\left(K_{b}\right)$ approaches.

under certain boundary conditions, we can obtain the dissipation values at the element size, in the actual domain, $e(x)$. Now, considering again the total domain divided into blocks, we can get dissipation values in a block, $\bar{e}(\mathbf{x}, V)$ (implying that $\bar{e}$ depends both on location and block size), by simply integrating $e(x)$ over the block. We can, then, obtain the mean, $\bar{e}_{m}$, and variance, $s_{\bar{e}}^{2}$, which can be considered good estimators of $\langle\bar{e}\rangle$ and $\sigma_{\bar{e}}^{2}$. These will be our reference values. Any methodology should give values close to these in order to verify the "necessary conditions" for the "sufficient" ones. Then, our reference option (option 1 ) to compute $\bar{e}$ will be

$$
\bar{e}_{1}(V)=\sum_{i=1}^{n_{e}} \nabla h_{i} \cdot K_{t} \cdot \nabla h_{i}
$$

where $n_{e}$ is number of elements in a block (in our particular example equal to 100), $K_{\imath}$ is conductivity of element $i$, and $\nabla h_{i}$ is head gradient, considered constant throughout the element.

The next step is to analyze the two different approaches of upscaling cited previously. Therefore our second option for computing $\bar{e}(\mathbf{x}, V)$ is based on using $K_{b}$ values as proposed by Rubin and Gómez-Hernández [1990]. Then, we replace the original $K$ field by the upscaled one and again solve the flow problem in the upscaled medium. To avoid numerical differences between this option and the previous one, we divided each block into 100 squared elements (reproducing the mesh at the local scale), but now assigned to each element a value equal to $K_{b}$. We have again a $420 \times 420$ element domain, but only $42 \times 42$ different values. The new head values will be denoted by $h^{b}(\mathbf{x})$. Option 2 will then be to compute $\bar{e}$ as

$$
\bar{e}_{2}(V)=\sum_{\imath=1}^{n_{e}} \nabla h_{\imath}^{b} \cdot K_{b} \cdot \nabla h_{\imath}^{b}=K_{b} \sum_{\imath=1}^{n_{e}} \nabla h_{i}^{b} \cdot \nabla h_{\imath}^{b}
$$

where $\nabla h_{l}^{b}$ are computed at the element size.

The third option is based on the Darcian approach. Hence we compute block conductivities, $K_{\text {exx }}$, and repeat the procedure explained above, i.e., replace the actual by the upscaled medium and again solve the flow problem under the same boundary conditions. Head values obtained from this new field will be denoted by $h^{e}(\mathbf{x})$, so that option 3 becomes 
Table 2. Comparison of Mean and Variance Values of Block Dissipation

\begin{tabular}{lccc}
\hline \multicolumn{1}{c}{ Option } & $e_{b, m} \times 10^{-2}$ & $s_{e_{b, m}}^{2} \times 10^{-3}$ & $\begin{array}{c}\text { Correlation } \\
\text { Factor }(r)\end{array}$ \\
\hline 1 (reference) & 1.275 & 4.07 & \\
2 & 1.281 & 3.70 & 0.967 \\
3 & 1.277 & 3.74 & 0.967 \\
4 & 1.285 & 4.16 & 0.995 \\
Theoretical & 1.273 & $\cdots$ & \\
\hline
\end{tabular}

$$
\overline{\boldsymbol{e}}_{3}(V)=\sum_{\imath=1}^{n_{e}} \nabla h_{\imath}^{e} \cdot K_{e x x} \cdot \nabla h_{\imath}^{e}=K_{\text {exx }} \sum_{\imath=1}^{n_{e}} \nabla h_{\imath}^{e} \cdot \nabla h_{\imath}^{e}
$$

The two previous options have in common that flow must be solved again and that head gradients are computed at the element size.

The last option we want to consider is a somewhat mixed approach. It does not require one to solve the flow problem again, but takes the head values from the original field, $h(\mathbf{x})$. Then, option 4 to compute energies is

$$
\bar{e}_{4}(V)=n_{e} \times\left(\overline{\nabla h} \cdot K_{b} \cdot \overline{\nabla h}\right)
$$

where again $\nabla h$ are computed at the block size (similar to option 1). Although this last option may seem cumbersome, it is easy to ascertain that it gives the exact results both for blocks with size corresponding to the element, where $\bar{e}_{4}=$ $\nabla h \cdot K \cdot \nabla h=\bar{e}_{1}$, and for very large blocks, where $\bar{e}_{4}=$ $K_{\text {eff }} \overline{\nabla h} \cdot \overline{\nabla h}=K_{\text {eff }}\left\langle j_{1}\right\rangle^{2}$, which is the exact expression for $\langle e\rangle$, as given in Appendix B, equation (B12). For intermediate block sizes, we show in Appendix B that by considering small perturbations, the energy function provides the same first two moments as option 1, i.e., $\left\langle\bar{e}_{1}\right\rangle=\left\langle\bar{e}_{4}\right\rangle$ and $C_{\bar{e}_{1}}=C_{\bar{e}_{4}}$, at least up to first order in $\sigma_{Y}^{2}$.

Statistical moments of block dissipation are summarized in Table 2, where the mean value of block dissipation $\left(e_{b, m}\right)$, as well as the variance $\left(s_{e_{b, m}}^{2}\right)$, is displayed. It can be seen that mean values agree very well with theory, with differences that never exceed $1 \%$ of the reference value. In the same table we also present the theoretical value, which is given, for example, in (B12) and (B19).

Variances also behave quite well with respect to the reference value. In general, it can be seen that options 2 and 3 give a somewhat smaller value $(\simeq 10 \%$ smaller), while option 4 gives a closer one ( $\approx 3 \%$ error). We have to keep in mind that $\sigma_{Y}^{2}=1.0$, far from the small-perturbation hypothesis, so that the error in this last case can be considered acceptable. Another way to check agreement between the different options is to directly compare block energies. For this purpose, we plot in Figure 11 block energy values for the reference case with respect to the three other options: 2,3 , and 4 , respectively. The 1:1 lines are also plotted for comparison purposes. Remember that a perfect correlation here is what Indelman and Dagan call "sufficient" conditions. We see again that the best results are obtained with option 4 .

\section{Summary and Conclusions}

Different approaches to upscaling of hydraulic conductivity in isotropic heterogeneous media have been studied for the case of square or cubic upscaling blocks. A literature review provided at least three practical upscaling approaches and a general methodology giving a number of conditions that any of them should verify. The three practical methods include a Darcian type approach (basically reproducing Darcy's experiment), power averaging (which reduces to geometric averaging for two dimensions), and defining block conductivity as the ratio of average flux to average gradient. On the other hand,
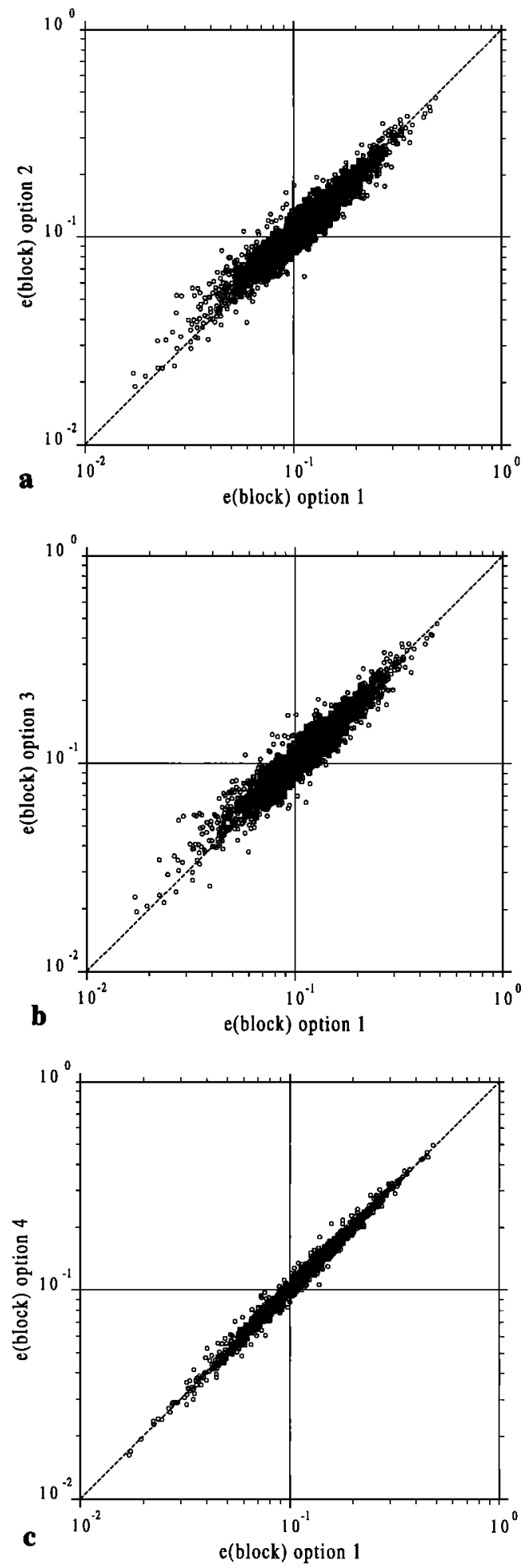

Figure 11. Comparison between block energy values given by (a) option 2, (b) option 3, and (c) option 4 with respect to the reference values (option 1 ). 
Indelman and Dagan [1993a] argued that upscaling should be based on preserving dissipation energy in the blocks.

Our work has been motivated by conjecturing that all these approaches are indeed very similar. Such a conjecture is based both on numerical simulations (Desbarats [1992] in three dimensions and Fenton and Griffiths [1993] in two dimensions) and on the analysis of limiting cases, as discussed in section 2. The method of Rubin and Gómez-Hernández [1990] is identical to the Darcian one when parallel flow is imposed at the block scale (boundary conditions of Figure 1c). Under these conditions, dissipation energy is preserved. In this case, as block conductivities are independent of flow conditions (a "desirable" condition), those approaches are indeed the appropriate ones.

With this motivation in mind, our work can be divided in two parts (somewhat mixed throughout the paper for brevity): first, verifying that the three "practical" approaches are very similar and, second, showing that all of them preserve, approximately, dissipation energy. For the first objective, we have derived the statistics of $Y_{b}$ (equal to $\ln K_{b}, K_{b}$ being computed by Rubin and Gómez-Hernández's method). By means of a smallperturbation approach, we were able to find analytically the first two moments of block log conductivity, considered as a random function. In the two-dimensional case we found $\left\langle Y_{b}\right\rangle$ $=\langle Y\rangle$, independent of block size, while in three dimensions, $\left\langle Y_{b}\right\rangle=\langle Y\rangle+\frac{1}{6}(1-\alpha) \sigma_{Y}^{2}$. The variance is given by $\sigma_{Y_{b}}^{2}=$ $\alpha \sigma_{Y}^{2}$, where $\alpha$ is a function of normalized block size (block size divided by integral scale), variogram model, and dimensionality; $\alpha$ is easily obtained by a numerical quadrature. These formulas coincide with the ones given empirically by Desbarats [1992] and by Fenton and Griffiths [1993].

Comparison between the Darcian and Rubin and GómezHernández's approaches was performed numerically. We found them to give very similar values (even more so in statistical terms), at least up to $\sigma_{Y}^{2}=1.0$ (Figure 10).

Validation of the three "practical" approaches with the proposal of Indelman and Dagan [1993a, b] is more difficult because these authors did not provide any methodology for computing actual block conductivities from point conductivities. Hence we have checked that the "practical" approaches indeed satisfy the conditions suggested by these authors, which are of two types. First, to ensure that the statistics of fluxes are kept, it is necessary that $K_{\text {eff }}=\widetilde{K}_{\text {eff }}$ and it is sufficient that the mean and covariance function of the block dissipation energies are identical in the actual and upscaled fields. Second, to ensure that head statistics are also kept, the actual spatial distribution of block dissipation energies must be close in the actual and upscaled fields. We have shown that the "practical" methods meet these requirements in a satisfactory way.

For one thing, we have shown analytically, under small perturbations, that $K_{\text {eff }}$ derived from the statistics of $Y_{b}$ is identical to that derived from the statistics of $Y$, both for two and three dimensions. This result was verified numerically for the twodimensional case. We should stress that Indelman and Dagan [1993a] stated the opposite, arguing that Rubin and GómezHernández's [1990] method did not ensure $K_{\text {eff }}=\bar{K}_{\text {eff }}$. In fact, this equality is only true if $K_{b}$ is taken as lognormally distributed. Lognormality of $K_{b}$ was proved numerically in a twodimensional example.

Deriving the mean and covariance function of blockaveraged dissipation $(\bar{e})$ when using the "practical" methods of upscaling is not easy. For the purpose of theoretical derivations, we were forced to approximate head gradients computed with upscaled permeabilities by block-averaged values of the true gradients. With this simplification we have proven that the mean and covariance function of the true $\bar{e}$ are identical to those obtained with $K_{b}$ (Appendix B).

Furthermore, we checked numerically that the blockaveraged dissipation computed by the practical methods is indeed very close to the true value in each block (Figure 11).

Some important conclusions can be stated from these results. First, all upscaling approaches studied in this work seem to provide similar results for block conductivity values, agreement that is striking in statistical terms. Second, we have found that at least up to $\sigma_{Y}^{2}=1.0$, simple approaches to upscaling that consider block $K$ as scalar give results which can compete with the general approach, where $K_{b}$ is treated as a tensor (probably, the second conclusion is only true for rather small variances). These are, indeed, good news because, first, the methodologies are simple and, second, they allow us to derive theoretical results. It should be stressed, however, that treating block $K$ as a scalar may not be valid for complex flow fields or for larger variances. Certainly, tensorial block $K$ are required for elongated blocks.

Finally, we should stress that one of the most important motivations of upscaling is conditioning block values upon measurements taken at different supports. At this point, it is important to remember that only Rubin and GómezHernández's formulation, working either with $K_{b}$ [GómezHernández, 1991] or with $Y_{b}$, as we propose, can handle conditioning in a direct way.

\section{Appendix A: Relationship Between $\overline{C_{Y_{j}}}$ and $\overline{C_{Y}}$}

In this appendix we want to derive (35) analytically. The starting point is the relationship between $C_{Y h}\left(\mathbf{x}, \mathbf{x}^{\prime}\right)$ and $C_{Y}(\mathbf{x}$, $\left.\mathbf{x}^{\prime}\right)$ given, for example, by Dagan [1989, equation 3.3.12]:

$$
\nabla_{x^{\prime}}^{2} C_{Y h}\left(\mathbf{x}, \mathbf{x}^{\prime}\right)=-\mathbf{j} \nabla_{x^{\prime}} C_{Y}\left(\mathbf{x}, \mathbf{x}^{\prime}\right)
$$

Note that in our formula a minus sign appears, due to the difference between Dagan's definition of $\mathbf{j}=-\nabla h$, and ours, $\mathbf{j}=\nabla h$.

In the simple case analyzed, $\mathbf{j}=\left(j_{1}, 0,0\right)$, so (A1) can be rewritten as

$$
\nabla_{x^{\prime}}^{2} C_{Y h}\left(\mathbf{x}, \mathbf{x}^{\prime}\right)=-j_{1} \frac{\partial}{\partial x^{\prime}} C_{Y}\left(\mathbf{x}, \mathbf{x}^{\prime}\right)
$$

This is a Poisson type equation which can be solved with the aid of Green's functions, so that

$$
C_{Y h}\left(\mathbf{x}, \mathbf{x}^{\prime}\right)=j_{1} \int_{\Omega} \frac{\partial}{\partial x^{\prime \prime}} C_{Y}\left(\mathbf{x}, \mathbf{x}^{\prime \prime}\right) G\left(\mathbf{x}^{\prime}, \mathbf{x}^{\prime \prime}\right) d \mathbf{x}^{\prime \prime}
$$

where $\Omega$ is an infinite domain; integration by parts leads to

$$
C_{Y h}\left(\mathbf{x}, \mathbf{x}^{\prime}\right)=-j_{1} \int_{\Omega} C_{Y}\left(\mathbf{x}, \mathbf{x}^{\prime \prime}\right) \frac{\partial}{\partial x^{\prime \prime}} G\left(\mathbf{x}^{\prime}, \mathbf{x}^{\prime \prime}\right) d \mathbf{x}^{\prime \prime}
$$

We can now use (24) to compute $C_{Y_{J_{1}}}\left(\mathbf{x}, \mathbf{x}^{\prime}\right)$ :

$$
\begin{aligned}
C_{Y_{j 1}}\left(\mathbf{x}, \mathbf{x}^{\prime}\right) & =\frac{\partial}{\partial x^{\prime}} C_{Y h}\left(\mathbf{x}, \mathbf{x}^{\prime}\right) \\
& =-j_{1} \int_{\Omega} C_{Y}\left(\mathbf{x}, \mathbf{x}^{\prime \prime}\right) \frac{\partial}{\partial x^{\prime}} \frac{\partial}{\partial x^{\prime \prime}} G\left(\mathbf{x}^{\prime}, \mathbf{x}^{\prime \prime}\right) d \mathbf{x}^{\prime \prime}
\end{aligned}
$$


If we look at the expression of $G\left(\mathbf{x}^{\prime}, \mathbf{x}^{\prime \prime}\right)$ in an unbounded domain, we see that

$$
G\left(\mathbf{x}^{\prime}, \mathbf{x}^{\prime \prime}\right)=f\left(\left|\mathbf{x}^{\prime}-\mathbf{x}^{\prime \prime}\right|\right)
$$

so that

$$
\begin{aligned}
\frac{\partial}{\partial x^{\prime}} \frac{\partial}{x^{\prime \prime}} G\left(\mathbf{x}^{\prime}, \mathbf{x}^{\prime \prime}\right)= & \frac{\partial}{\partial x^{\prime}} \frac{\partial}{\partial x^{\prime \prime}} f\left(\left|\mathbf{x}^{\prime}-\mathbf{x}^{\prime \prime}\right|\right) \\
& =\frac{\partial}{\partial x^{\prime}}\left(f^{\prime}\left(\left|\mathbf{x}^{\prime}-\mathbf{x}^{\prime \prime}\right|\right) \frac{x^{\prime \prime}-x^{\prime}}{\left|\mathbf{x}^{\prime}-\mathbf{x}^{\prime \prime}\right|}\right) \\
& =-f^{\prime \prime}\left(\left|\mathbf{x}^{\prime}-\mathbf{x}^{\prime \prime}\right|\right) \frac{\left(x^{\prime}-x^{\prime \prime}\right)^{2}}{\left|\mathbf{x}^{\prime}-\mathbf{x}^{\prime \prime}\right|^{2}}-\frac{f^{\prime}\left(\left|\mathbf{x}^{\prime}-\mathbf{x}^{\prime \prime}\right|\right)}{\left|\mathbf{x}^{\prime}-\mathbf{x}^{\prime \prime}\right|^{2}} \\
& \cdot\left(\left|\mathbf{x}^{\prime}-\mathbf{x}^{\prime \prime}\right|-\frac{\left(x^{\prime}-x^{\prime \prime}\right)^{2}}{\left|\mathbf{x}^{\prime}-\mathbf{x}^{\prime \prime}\right|}\right)
\end{aligned}
$$

while

$$
\begin{aligned}
\frac{\partial^{2}}{\partial x^{\prime \prime}} G\left(\mathbf{x}^{\prime}, \mathbf{x}^{\prime \prime}\right)= & \frac{\partial}{\partial x^{\prime \prime}}\left(f^{\prime}\left(\left|\mathbf{x}^{\prime}-\mathbf{x}^{\prime \prime}\right|\right) \frac{x^{\prime \prime}-x^{\prime}}{\left|\mathbf{x}^{\prime}-\mathbf{x}^{\prime \prime}\right|}\right) \\
= & f^{\prime \prime}\left(\left|\mathbf{x}^{\prime}-\mathbf{x}^{\prime \prime}\right|\right) \frac{\left(x^{\prime}-x^{\prime \prime}\right)^{2}}{\left|\mathbf{x}^{\prime}-\mathbf{x}^{\prime \prime}\right|^{2}}+\frac{f^{\prime}\left(\left|\mathbf{x}^{\prime}-\mathbf{x}^{\prime \prime}\right|\right)}{\left|\mathbf{x}^{\prime}-\mathbf{x}^{\prime \prime}\right|^{2}} \\
& \cdot\left(\left|\mathbf{x}^{\prime}-\mathbf{x}^{\prime \prime}\right|-\frac{\left(x^{\prime}-x^{\prime \prime}\right)^{2}}{\left|\mathbf{x}^{\prime}-\mathbf{x}^{\prime \prime}\right|}\right)
\end{aligned}
$$

so, we can rewrite (A5) as

$$
C_{Y_{11}}\left(\mathbf{x}, \mathbf{x}^{\prime}\right)=j_{1} \int_{\Omega} C_{Y}\left(\mathbf{x}, \mathbf{x}^{\prime \prime}\right) \frac{\partial^{2}}{\partial x^{\prime \prime 2}} G\left(\mathbf{x}^{\prime}, \mathbf{x}^{\prime \prime}\right) d \mathbf{x}^{\prime \prime}
$$

which will prove to be a very useful expression in our next derivations.

Now, what we are really interested in is computing the spatial average of (A9); i.e., we want to get

$$
\begin{aligned}
\overline{C_{Y j 1}}= & \frac{1}{V^{2}} \int_{V} \int_{V} C_{Y_{j 1}}\left(\mathbf{x}, \mathbf{x}^{\prime}\right) d \mathbf{x} d \mathbf{x}^{\prime} \\
= & j_{1} \frac{1}{V^{2}} \int_{V} \int_{V} \int_{\Omega} C_{Y}\left(\mathbf{x}, \mathbf{x}^{\prime \prime}\right) \\
& \cdot \frac{\partial^{2}}{\partial x^{\prime \prime 2}} G\left(\mathbf{x}^{\prime}, \mathbf{x}^{\prime \prime}\right) d \mathbf{x}^{\prime \prime} d \mathbf{x} d \mathbf{x}^{\prime}
\end{aligned}
$$

This can be expanded using (A8) and assuming $C_{Y}\left(\mathbf{x}, \mathbf{x}^{\prime \prime}\right)=$ $g\left(\left|\mathbf{x}-\mathbf{x}^{\prime \prime}\right|\right)$ :

$$
\begin{aligned}
\overline{C_{Y_{11}}}= & j_{1} \frac{1}{V^{2}} \int_{V} \int_{V} \int_{\Omega} g\left(\left|\mathbf{x}-\mathbf{x}^{\prime \prime}\right|\right) \\
& \cdot\left[f^{\prime \prime}\left(\left|\mathbf{x}^{\prime}-\mathbf{x}^{\prime \prime}\right|\right) \frac{\left(x^{\prime}-x^{\prime \prime}\right)^{2}}{\left|\mathbf{x}^{\prime}-\mathbf{x}^{\prime \prime}\right|^{2}}+\frac{f^{\prime}\left(\left|\mathbf{x}^{\prime}-\mathbf{x}^{\prime \prime}\right|\right)}{\left|\mathbf{x}^{\prime}-\mathbf{x}^{\prime \prime}\right|^{2}}\right. \\
& \left.\cdot\left(\left|\mathbf{x}^{\prime}-\mathbf{x}^{\prime \prime}\right|-\frac{\left(x^{\prime}-x^{\prime \prime}\right)^{2}}{\left|\mathbf{x}^{\prime}-\mathbf{x}^{\prime \prime}\right|}\right)\right] d \mathbf{x}^{\prime \prime} d \mathbf{x} d \mathbf{x}^{\prime}
\end{aligned}
$$

Let us consider now the case of $V$ being a parallelepiped with equal sides (i.e., a square in two dimensions or a cube in three dimensions). For this particular case, simple inspection of (A11) allows us to conclude that the same value for the $n$-tuple integral will be obtained if we simply replace $\left(x^{\prime}-x^{\prime \prime}\right)$ by $\left(y^{\prime}-y^{\prime \prime}\right)$ or $\left(z^{\prime}-z^{\prime \prime}\right)$. This is so because of the symmetry of the integrand and because the integration domain remains unchanged. This means that under, and only under, this condition the second partial derivative $\partial^{2} / \partial x^{\prime 2}$ in (A9) can be perfectly exchanged with $\partial^{2} / \partial y^{\prime \prime 2}$ or $\partial^{2} / \partial z^{\prime \prime}$. This allows us to conclude

$\overline{C_{Y_{1}}}=j_{1} \frac{1}{V^{2}} \int_{V} \int_{V} \int_{\Omega} C_{Y}\left(\mathbf{x}, \mathbf{x}^{\prime \prime}\right) \frac{1}{n} \nabla_{x^{\prime \prime}}^{2} G\left(\mathbf{x}^{\prime}, \mathbf{x}^{\prime \prime}\right) d \mathbf{x}^{\prime \prime} d \mathbf{x} d \mathbf{x}^{\prime}$

where $n$ is the dimensionality of the problem. And again, using Green's function properties, we can find the final expression:

$$
\begin{aligned}
\overline{C_{Y_{\jmath 1}}} & =-j_{1} \frac{1}{V^{2}} \int_{V} \int_{V} \int_{\Omega} C_{Y}\left(\mathbf{x}, \mathbf{x}^{\prime \prime}\right) \frac{1}{n} \delta\left(\mathbf{x}^{\prime}-\mathbf{x}^{\prime \prime}\right) d \mathbf{x}^{\prime \prime} d \mathbf{x} d \mathbf{x}^{\prime} \\
& =-j_{1} \frac{1}{V^{2}} \frac{1}{n} \int_{V} \int_{V} C_{Y}\left(\mathbf{x}, \mathbf{x}^{\prime}\right) d \mathbf{x} d \mathbf{x}^{\prime}=\frac{-j_{1}}{n} \overline{C_{Y}}
\end{aligned}
$$

where $\delta(\quad)$ is the Dirac delta function. This last expression is (35), which we were seeking.

\section{Appendix B: Check on "Sufficient" Conditions for Option 4}

Our starting point is the definition of the dissipation function $e(\mathbf{x})$, as given by Indelman and Dagan [1993a], written, using the variables in this paper, as

$$
e(\mathbf{x})=\nabla h^{t} \cdot K \cdot \nabla h
$$

We want to check an alternative to computing $e(\mathbf{x})$ in a block:

$$
\hat{e}(\mathbf{x})=\overline{\nabla h^{\iota}} \cdot K_{b} \cdot \overline{\nabla h}
$$

referred to as option 4 in the text. We want to prove that this formulation verifies what Indelman and Dagan [1993a] note as sufficient conditions:

$$
\langle e(\mathbf{x})\rangle=\langle\hat{e}(\mathbf{x})\rangle \quad C_{\bar{e}}(\mathbf{r})=C_{\hat{e}}(\mathbf{r})
$$

By using a small-perturbation expansion it is easy to show [Indelman and Dagan, 1993b] that $e(\mathbf{x})$ can be written, using the same variables that appear in the text, as

$$
\begin{aligned}
& \langle e\rangle=K_{G}\left[\left(1+\frac{\sigma_{Y}^{2}}{2}\right)\langle j\rangle^{2}+2\langle\mathbf{j}\rangle \cdot\left\langle Y^{\prime} \nabla h^{\prime}\right\rangle+\left\langle\nabla h^{\prime} \cdot \nabla h^{\prime}\right\rangle\right] \\
& e^{\prime}(\mathbf{x})=K_{G}\left[Y^{\prime}\langle j\rangle^{2}+2\langle\mathbf{j}\rangle \cdot \nabla h^{\prime}\right]=K_{G}\left[Y^{\prime}\left\langle j_{1}\right\rangle^{2}+2\left\langle j_{1}\right\rangle j_{1}^{\prime}\right] \\
& C_{e}(\mathbf{r})=K_{G}^{2}\left[\left\langle j_{1}\right\rangle^{4} C_{Y}(\mathbf{r})+4\left\langle j_{1}\right\rangle^{3} C_{Y_{j_{1}}}(\mathbf{r})+4\left\langle j_{1}\right\rangle^{2} C_{j J_{1}}(\mathbf{r})\right]
\end{aligned}
$$

We have seen in the text that $\left\langle Y^{\prime}\left(\partial h^{\prime} / \partial x_{1}\right)\right\rangle=-\left(\sigma_{Y}^{2} / n\right)\left\langle j_{1}\right\rangle$; now we want to evaluate $\left\langle\nabla h^{\prime}(\mathbf{x}) \cdot \nabla h^{\prime}(\mathbf{x})\right\rangle$. For that purpose we can use equation 1.4.15 of Dagan [1989], 


$$
A_{h}=\left\langle\nabla h^{\prime}(\mathbf{x}) \cdot \nabla h^{\prime}(\mathbf{x})\right\rangle=-\left[\nabla^{2} C_{h}(\mathbf{r})\right]_{\mathrm{r}=0}
$$

and using the Fourier transform (FT) technique,

$$
A_{h}=\left.\frac{1}{(2 \pi)^{n / 2}} \int k^{2} \hat{C}_{h}(\mathbf{k}) e^{-\mathbf{k r} r} d \mathbf{k}\right|_{\mathbf{r}=0}
$$

with $k=|\mathbf{k}|$, where $\mathbf{k}$ is the wave number vector. Now, using equation 3.7.13 of Dagan [1989], we can express it by the FT of $C_{Y}(\mathbf{r})$, and taking $\mathbf{r}=0$, we have

$$
A_{h}=\frac{\left\langle j_{1}\right\rangle^{2}}{(2 \pi)^{n / 2}} \int \frac{k_{1}^{2}}{k^{2}} \hat{C}_{Y}(\mathbf{k}) d \mathbf{k}
$$

Now we have to distinguish the two- and three-dimensional cases; in two dimensions we switch to polar coordinates $\left(k_{1}=\right.$ $k \cos \theta, k_{2}=k \sin \theta$ ), and we get

$$
\begin{aligned}
A_{h, 2} & =\frac{\left\langle j_{1}\right\rangle^{2}}{2 \pi} \int_{0}^{\infty} \int_{0}^{2 \pi} \cos ^{2} \theta \hat{C}_{Y}(k) k d k d \theta \\
& =\frac{\left\langle j_{1}\right\rangle^{2}}{2} \int_{0}^{\infty} k \hat{C}_{Y}(k) d k=\frac{\left\langle j_{1}\right\rangle^{2}}{2} \sigma_{Y}^{2} \int_{0}^{\infty} \frac{k I^{2}}{\left(1+k^{2} I^{2}\right)^{3 / 2}} d k \\
& =\frac{\left\langle j_{1}\right\rangle^{2} \sigma_{Y}^{2}}{2}
\end{aligned}
$$

In the three-dimensional case, instead, we switch to spherical coordinates $\left(k_{1}=k \sin \theta \cos \phi, k_{2}=k \sin \theta \sin \phi, k_{3}=k\right.$ $\cos \theta)$, and we get

$$
\begin{aligned}
A_{h, 3} & =\frac{\left\langle j_{1}\right\rangle^{2}}{(2 \pi)^{3 / 2}} \int_{0}^{\infty} \int_{0}^{\pi} \int_{0}^{2 \pi} \sin ^{3} \theta \cos ^{2} \phi \hat{C}_{Y}(k) k^{2} d \phi d \theta d k \\
& =\frac{\left\langle j_{1}\right\rangle^{2}}{(2 \pi)^{3 / 2}} \frac{4 \pi}{3} \int_{0}^{\infty} \hat{C}_{Y}(k) k^{2} d k=\frac{\left\langle j_{1}\right\rangle^{2}}{(2 \pi)^{1 / 2}} \frac{2}{3}\left(\frac{\pi}{2}\right)^{1 / 2} \sigma_{Y}^{2} \\
& =\frac{\left\langle j_{1}\right\rangle^{2} \sigma_{Y}^{2}}{3}
\end{aligned}
$$

So, in general, $A_{h, n}=\left\langle j_{1}\right\rangle^{2} \sigma_{Y}^{2} / n$ for $n=2,3$; thus we get our final expression for $\langle e\rangle$ in an isotropic medium

$$
\langle e\rangle=K_{G}\left\langle j_{1}\right\rangle^{2}\left[1+\left(\frac{1}{2}-\frac{1}{n}\right) \sigma_{Y}^{2}\right]=K_{\mathrm{eff}}\left\langle j_{1}\right\rangle^{2}
$$

Our next step is to derive $\langle\hat{e}\rangle$ based on (B2), again by using small-perturbation expansion (similar to (16) and (18)),

$$
\begin{aligned}
\hat{e}= & \overline{\nabla h^{\prime}} K_{b} \overline{\nabla h}=\overline{\mathbf{q}} \cdot \overline{\mathbf{j}}=\overline{K\left(\langle\mathbf{j}\rangle+\mathbf{j}^{\prime}\right)} \cdot \overline{\langle\mathbf{j}\rangle+\overline{\mathbf{j}}^{\prime}} \\
= & K_{G}\left[\langle\mathbf{j}\rangle^{2}+\langle\mathbf{j}\rangle^{2} Y^{\prime}+\langle\mathbf{j}\rangle^{2} \frac{Y^{\prime 2}}{2}+2\langle\mathbf{j}\rangle \cdot \overline{\mathbf{j}^{\prime}}+\langle\mathbf{j}\rangle \cdot \overline{\mathbf{j}^{\prime} \bar{Y}^{\prime}}\right. \\
& \left.+\langle\mathbf{j}\rangle \cdot \overline{\mathbf{j}^{\prime}} \overline{Y^{\prime}}+\overline{\mathbf{j}^{\prime}} \cdot \overline{\mathbf{j}^{\prime}}\right]
\end{aligned}
$$

and as $\langle\mathbf{j}\rangle=\left(\left\langle j_{1}\right\rangle, 0,0\right)$ and taking the expected value,

$$
\begin{aligned}
\langle\hat{e}\rangle= & K_{G}\left[\left\langle j_{1}\right\rangle^{2}\left(1+\left\langle\bar{Y}^{\prime}\right\rangle+\left\langle\overline{Y^{\prime 2}} \overline{2}\right\rangle\right)+2\left\langle j_{1}\right\rangle\left\langle\overline{j_{1}^{\prime}}\right\rangle+\left\langle j_{1}\right\rangle\right. \\
& \left.\cdot\left(\left\langle\overline{j_{1}^{\prime} Y^{\prime}}\right\rangle+\left\langle\overline{j_{1}^{\prime}} \bar{Y}^{\prime}\right\rangle\right)+\left\langle\overline{j_{1}^{\prime}} \overline{j_{1}^{\prime}}\right\rangle+\left\langle\overline{j_{2}^{\prime}} \overline{j_{2}^{\prime}}\right\rangle\right] \\
= & K_{G}\left[\left\langle j_{1}\right\rangle^{2}\left(1+\frac{\sigma_{Y}^{2}}{2}\right)+\left\langle j_{1}\right\rangle\left(C_{Y_{j 1}}(0)+\overline{C_{Y j 1}}\right)\right. \\
& \left.+\overline{C_{J_{1} / 1}}+\overline{C_{J_{2} / 2}}\right]
\end{aligned}
$$

Using the properties of differentiation of random functions, we know that

$$
\overline{C_{11 / 1}}+\overline{C_{j 2 / 2}}=-\frac{\overline{\partial^{2} C_{h h}}}{\partial x_{1}^{2}}-\frac{\overline{\partial^{2} C_{h h}}}{\partial x_{2}^{2}}=-\overline{\nabla^{2} C_{h h}}
$$

where [Dagan, 1989, equation 3.3.13]

$$
\nabla_{2}^{2} \nabla_{1}^{2} C_{h h}\left(\mathbf{x}_{1}, \mathbf{x}_{2}\right)=-\left\langle j_{1}\right\rangle^{2} \frac{\partial^{2}}{\partial x_{1}^{2}} C_{Y}\left(\mathbf{x}_{1}, \mathbf{x}_{2}\right)
$$

Solving this equation with the aid of Green's function,

$$
\begin{aligned}
\overline{\nabla^{2} C_{h h}} & =\frac{1}{V^{2}} \int_{V} \int_{V} \nabla^{2} C_{h h}\left(\mathbf{x}_{1}, \mathbf{x}_{2}\right) d \mathbf{x}_{2} d \mathbf{x}_{1} \\
& =\frac{\left\langle j_{1}\right\rangle^{2}}{V^{2}} \int_{V} \int_{V} \int_{\Omega} \frac{\partial^{2}}{\partial x_{3}^{2}} C_{Y}\left(\mathbf{x}_{3}, \mathbf{x}_{2}\right) G\left(\mathbf{x}_{1}, \mathbf{x}_{3}\right) d \mathbf{x}_{3} d \mathbf{x}_{2} d \mathbf{x}_{1} \\
& =\frac{\left\langle j_{1}\right\rangle^{2}}{V^{2}} \int_{V} \int_{V} \int_{\Omega} C_{Y}\left(\mathbf{x}_{3}, \mathbf{x}_{2}\right) \frac{\partial^{2}}{\partial x_{3}^{2}} G\left(\mathbf{x}_{1}, \mathbf{x}_{3}\right) d \mathbf{x}_{3} d \mathbf{x}_{2} d \mathbf{x}_{1}
\end{aligned}
$$

and again using symmetry considerations (as in Appendix A), we get

$$
\begin{aligned}
\overline{\nabla^{2} C_{h h}} & =\frac{\left\langle j_{1}\right\rangle^{2}}{V^{2}} \int_{V} \int_{V} \int_{\Omega} C_{Y}\left(\mathbf{x}_{3}, \mathbf{x}_{2}\right) \frac{1}{n} \nabla_{x_{3}}^{2} G\left(\mathbf{x}_{1}, \mathbf{x}_{3}\right) d \mathbf{x}_{3} d \mathbf{x}_{2} d \mathbf{x}_{1} \\
= & -\frac{\left\langle j_{1}\right\rangle^{2}}{V^{2}} \int_{V} \int_{V} \int_{\Omega} C_{Y}\left(\mathbf{x}_{3}, \mathbf{x}_{2}\right) \\
& \cdot \frac{1}{n} \delta\left(\mathbf{x}_{1}-\mathbf{x}_{3}\right) d \mathbf{x}_{3} d \mathbf{x}_{2} d \mathbf{x}_{1}=-\frac{\left\langle j_{1}\right\rangle^{2}}{n} \overline{C_{Y}}
\end{aligned}
$$

So, we can get our final expression,

$$
\begin{aligned}
\langle\hat{e}\rangle & =K_{G}\left\langle j_{1}\right\rangle^{2}\left[1+\frac{\sigma_{Y}^{2}}{2}-\frac{\sigma_{Y}^{2}}{n}-\frac{\overline{C_{Y}}}{n}+\frac{\overline{C_{Y}}}{n}\right] \\
& =K_{G}\left\langle j_{1}\right\rangle^{2}\left[1+\sigma_{Y}^{2}\left(\frac{1}{2}-\frac{1}{n}\right)\right]=\langle e\rangle
\end{aligned}
$$

which is the first condition we were seeking. The rest of this appendix will be devoted to analyzing the condition on secondorder moments. For that purpose we have to keep in mind the definition of $\bar{e}(\mathbf{x})$ given by Indelman and Dagan [1993a, equation (6)], which in our notation is 


$$
\bar{e}(\mathbf{x})=\frac{1}{V} \int_{V(\mathbf{x})} e\left(\mathbf{x}^{\prime}\right) d \mathbf{x}^{\prime}
$$

so that

$$
\begin{gathered}
C_{\bar{e}}\left(\mathbf{x}, \mathbf{x}^{\prime}\right)=\frac{1}{V^{2}} \int_{V(\mathbf{x})} \int_{V\left(\mathbf{x}^{\prime}\right)}\left\langle e^{\prime}\left(\mathbf{x}^{\prime \prime}\right) e^{\prime}\left(\mathbf{x}^{\prime \prime \prime}\right)\right\rangle d \mathbf{x}^{\prime \prime} d \mathbf{x}^{\prime \prime \prime} \\
=\frac{1}{V^{2}} \int_{V(\mathbf{x})} \int_{V\left(\mathbf{x}^{\prime}\right)} C_{e}\left(\mathbf{x}^{\prime \prime}, \mathbf{x}^{\prime \prime \prime}\right) d \mathbf{x}^{\prime \prime} d \mathbf{x}^{\prime \prime \prime}=\overline{C_{e}}(\mathbf{r})
\end{gathered}
$$

with $\mathbf{r}=\mathbf{x}-\mathbf{x}^{\prime}$. By substituting in (B6), we get an expression for $C_{\bar{e}}$ up to first order in $\sigma_{Y}^{2}$ :

$C_{\bar{e}}=K_{G}^{2}\left[\left\langle j_{1}\right\rangle^{4} \overline{C_{Y}}(\mathbf{r})+4\left\langle j_{1}\right\rangle^{3} \overline{C_{Y_{j 1}}}(\mathbf{r})+4\left\langle j_{1}\right\rangle^{2} \overline{C_{j 11}}(\mathbf{r})\right]$

This value must be compared to $C_{\vec{e}}$. Our starting point is (B13) and (B14). By subtracting them, we get up to first order

$$
\hat{e}^{\prime}(\mathbf{x})=\hat{e}(\mathbf{x})-\langle\hat{e}\rangle=K_{G}\left\langle j_{1}\right\rangle^{2} \overline{Y^{\prime}}+2 K_{G}\left\langle j_{1}\right\rangle \overline{j_{1}^{\prime}}
$$

so that

$$
\begin{aligned}
C_{\hat{e}}= & \left\langle\hat{e}^{\prime}(\mathbf{x}) \hat{e}^{\prime}\left(\mathbf{x}^{\prime}\right)\right\rangle=K_{G}^{2}\left[\left\langle j_{1}\right\rangle^{4}\left\langle\overline{Y^{\prime}(\mathbf{x})} \overline{\left.Y^{\prime}\left(\mathbf{x}^{\prime}\right)\right\rangle}\right\rangle\right. \\
& +4\left\langle j_{1}\right\rangle^{3}\left\langle\overline{Y^{\prime}(\mathbf{x})} \overline{j_{1}^{\prime}\left(\mathbf{x}^{\prime}\right)}+4\left\langle j_{1}\right\rangle^{2}\left\langle\overline{j_{1}^{\prime}(\mathbf{x})} \overline{\left.j_{1}^{\prime}\left(\mathbf{x}^{\prime}\right)\right\rangle}\right]\right. \\
= & K_{G}^{2}\left[\left\langle j_{1}\right\rangle^{4} \overline{C_{Y}}(\mathbf{r})+4\left\langle j_{1}\right\rangle^{3} \overline{C_{Y_{j 1}}}(\mathbf{r})+4\left\langle j_{1}\right\rangle^{2} \overline{C_{j_{11}}}(\mathbf{r})\right]
\end{aligned}
$$

so we see up to first order in $\sigma_{Y}^{2}$

$$
C_{\bar{e}}(\mathbf{r})=C_{\hat{e}}(\mathbf{r})
$$

thus verifying the second sufficient condition.

Acknowledgments. This work was funded by ENRESA (Spanish nuclear waste disposal company) and the CEE through grant RADWAS F12W-CT91-0080 and by CICYT through grant PB-1015. Numerical work was performed on the supercomputer Cray YMP-2E at CESCA (Centre de Supercomputació de Catalunya). We want to thank G. Dagan, from Tel Aviv University (Israel), for suggesting the approach we used in the derivations in section 3 . We also want to thank an anonymous reviewer, whose helpful comments were incorporated in the final version of the paper.

\section{References}

Ababou, R., Effective conductivity tensor in randomly heterogeneous and anisotropic formations, paper presented at XVIII General Assembly, Eur. Geophys. Soc., Wiesbaden, Germany, 1993.

Ababou, R., and E. F. Wood, Comment on "Effective groundwater model parameter values: Influence of spatial variability of hydraulic conductivity, leakance, and recharge" by J. J. Gómez-Hernández and S. M. Gorelick, Water Resour. Res., 26(8), 1843-1846, 1990.

Dagan, G., Stochastic modeling of groundwater flow by unconditional and conditional probabilities, 1 , Conditional simulation and the direct problem, Water Resour. Res., 18(4), 813-833, 1982.

Dagan, G., Flow and Transport in Porous Formations, 465 pp., Springer-Verlag, New York, 1989.

Desbarats, A. J., Numerical estimation of effective permeability in sand-shale formations, Water Resour. Res., 23(2), 273-286, 1987.

Desbarats, A. J., Spatial averaging of hydraulic conductivity in threedimensional heterogeneous porous media, Math. Geol., 24(3), 249 267, 1992.

Durlofsky, L. J., Numerical calculation of equivalent grid block permeability tensors for heterogeneous porous media, Water Resour. Res., 27(5), 699-708, 1991.
Durlofsky, L. J., Representation of grid block permeability in coarse scale models of randomly heterogeneous porous media, Water Resour. Res., 28(7), 1791-1800, 1992.

Fenton, G. A., and D. V. Griffiths, Statistics of block conductivity through a simple bounded stochastic medium, Water Resour. Res., 29(6), 1825-1830, 1993.

Gelhar, L. W., and C. L. Axness, Three-dimensional stochastic analysis of macrodispersion in aquifers, Water Resour. Res., 19(1), 161-180, 1983.

Gómez-Hernández, J. J., Simulation of block permeabilities conditioned upon data measured at a different scale, in Proceedings, ModelCARE 90, LAHS Publ, 195, 407-416, 1990.

Gómez-Hernández, J. J., A Stochastic approach to the simulation of conductivity fields conditioned upon data measured at a smaller scale, Ph.D. Dissertation, Dep. of Appl. Sci., Stanford Univ., Stanford, Calif., 1991.

Gómez-Hernández, J. J., and S. M. Gorelick, Effective groundwater model parameter values: Influence of spatial variability of hydraulic conductivity, leakance, and recharge, Water Resour. Res., 25(3), 405419, 1989.

Gómez-Hernández, J. J., and A. G. Journel, Joint sequential simulation of multigaussian fields, in Geostatishcs Tróia '92, vol. 1, pp. 85-94, Kluwer Academic, Norwell, Mass., 1993.

Gutjahr, A. L., L. W. Gelhar, A. A. Bakr, and J. R. MacMillan, Stochastic analysis of spatial variability in subsurface flows, 2, Evaluation and application, Water Resour. Res., 14(5), 953-959, 1978.

Indelman, P., Upscaling of permeability of anisotropic formations, 3, Applications, Water Resour. Res., 29(4), 935-943, 1993.

Indelman, P., and G. Dagan, Upscaling of permeability of anisotropic heterogeneous formations, 1 , The general framework, Water Resour. Res., 29(4), 917-923, 1993a.

Indelman, P., and G. Dagan, Upscaling of permeability of anisotropic heterogeneous formations, 2, General structure and small perturbation analysis, Water Resour. Res., 29(4), 925-933, 1993 b.

Journel, A. G., C. Deutsch, and A. J. Desbarats, Power averaging for block effective permeability, paper SPE 15128 presented at the 56th California Regional Meeting of the Society of Petroleum Engineers, Oakland, Calif., 1986.

Kitanidis, P. K., Effective hydraulic conductivity for gradually varying flow, Water Resour. Res., 26(6), 1197-1208, 1990.

Loaiciga, H. A., R. B. Leipnik, M. A. Marin̄o, and P. F. Hudak, Stochastic groundwater flow analysis in the presence of trends in heterogeneous hydraulic conductivity fields, Math. Geol., 25(2), 161176,1993

Matheron, G., Eléments Pour une Théorie des Milieux Poreux, Masson et Cie, Paris, 1967.

Naff, R. L., Radial flow in heterogeneous porous media: An analysis of specific discharge, Water Resour. Res., 27(3), 307-316, 1991.

Neuman, S. P., and S. Orr, Prediction of steady state flow in nonuniform geologic media by conditional moments: Exact nonlocal formalism, effective conductivities and weak approximation, Water $R e$ sour. Res., 29(2), 341-364, 1993.

Rubin, Y., and J. J. Gómez-Hernández, A stochastic approach to the problem of upscaling of conductivity in disordered media: Theory and unconditional numerical simulations, Water Resour. Res., 26(4), 691-701, 1990.

Sánchez-Vila, X., I. Colominas, and J. Carrera, FAITH: User's guide, Univ. Politec. de Catalunya, Barcelona, Spain, 1993.

Schvidler, M. I., Filtration flows in heterogeneous media, Izv. Akad. Nauk SSSR Mekh. Mas., 3, 185-190, 1962.

Smith, L., and R. A. Freeze, Stochastic analysis of steady state groundwater flow in a bounded domain, 2, Two-dimensional simulations, Water Resour. Res., 15(6), 1543-1559, 1979.

J. Carrera, Departament d'Enginyeria del Terreny, Escola Tècnica Superior d'Enginyers de Camins, Universitat Politècnica de Catalunya, 08034 Barcelona, Spain.

J. P. Girardi, Consejo Nacional de Investigaciones Científicas y Técnicas, Universidad Nacional de San Juan, 5400 San Juan, Argentina.

X. Sánchez-Vila, Departament Matemàtica Aplicada III, Escola Tècnica Superior d'Enginyers de Camins, Universitat Politècnica de Catalunya, 08034 Barcelona, Spain.

(Received December 13, 1993; revised September 15, 1994; accepted October 20, 1994.) 